\title{
Diverse hydrogen production and consumption pathways influence methane production in ruminants
}

\author{
Chris Greening $\mathbb{1}^{1} \cdot$ Renae Geier $^{2} \cdot$ Cecilia Wang $^{3} \cdot$ Laura C. Woods $^{1} \cdot$ Sergio E. Morales ${ }^{3} \cdot$ Michael J. McDonald $^{1}$. \\ Rowena Rushton-Green ${ }^{3} \cdot$ Xochitl C. Morgan $^{3} \cdot$ Satoshi Koike $^{4} \cdot$ Sinead C. Leahy ${ }^{5}$. William J. Kelly ${ }^{6}$. Isaac Cann ${ }^{2}$. \\ Graeme T. Attwood ${ }^{5}$ Gregory M. Cook $^{3} \cdot$ Roderick I. Mackie ${ }^{2}$
}

Received: 7 December 2018 / Revised: 3 June 2019 / Accepted: 7 June 2019 / Published online: 26 June 2019

(c) The Author(s) 2019. This article is published with open access

\begin{abstract}
Farmed ruminants are the largest source of anthropogenic methane emissions globally. The methanogenic archaea responsible for these emissions use molecular hydrogen $\left(\mathrm{H}_{2}\right)$, produced during bacterial and eukaryotic carbohydrate fermentation, as their primary energy source. In this work, we used comparative genomic, metatranscriptomic and co-culture-based approaches to gain a system-wide understanding of the organisms and pathways responsible for ruminal $\mathrm{H}_{2}$ metabolism. Two-thirds of sequenced rumen bacterial and archaeal genomes encode enzymes that catalyse $\mathrm{H}_{2}$ production or consumption, including 26 distinct hydrogenase subgroups. Metatranscriptomic analysis confirmed that these hydrogenases are differentially expressed in sheep rumen. Electron-bifurcating [ $\mathrm{FeFe}$-hydrogenases from carbohydrate-fermenting Clostridia (e.g., Ruminococcus) accounted for half of all hydrogenase transcripts. Various $\mathrm{H}_{2}$ uptake pathways were also expressed, including methanogenesis (Methanobrevibacter), fumarate and nitrite reduction (Selenomonas), and acetogenesis (Blautia). Whereas methanogenesis-related transcripts predominated in high methane yield sheep, alternative uptake pathways were significantly upregulated in low methane yield sheep. Complementing these findings, we observed significant differential expression and activity of the hydrogenases of the hydrogenogenic cellulose fermenter Ruminococcus albus and the hydrogenotrophic fumarate reducer Wolinella succinogenes in co-culture compared with pure culture. We conclude that $\mathrm{H}_{2}$ metabolism is a more complex and widespread trait among rumen microorganisms than previously recognised. There is evidence that alternative hydrogenotrophs, including acetogenic and respiratory bacteria, can prosper in the rumen and effectively compete with methanogens for $\mathrm{H}_{2}$. These findings may help to inform ongoing strategies to mitigate methane emissions by increasing flux through alternative $\mathrm{H}_{2}$ uptake pathways, including through animal selection, dietary supplementation and methanogenesis inhibitors.
\end{abstract}

These authors contributed equally: Chris Greening and Renae Geier

Supplementary information The online version of this article (https:// doi.org/10.1038/s41396-019-0464-2) contains supplementary material, which is available to authorized users.

$\triangle$ Chris Greening

chris.greening@monash.edu

$\triangle$ Roderick I. Mackie

r-mackie@illinois.edu

1 School of Biological Sciences, Monash University, Clayton, VIC 3800 , Australia

2 Department of Animal Sciences and Institute for Genomic Biology, University of Illinois at Urbana-Champaign, Urbana, IL

\section{Introduction}

Methane production by livestock accounts for over 5\% of global greenhouse gas emissions annually [1]. These emissions mostly originate from the activity of methanogens within ruminants, which generate methane as an obligate

61801, USA

3 Department of Microbiology and Immunology, University of Otago, Dunedin 9016, New Zealand

4 Research Faculty of Agriculture, Hokkaido University, Sapporo, Japan

5 Grasslands Research Centre, AgResearch Ltd., Palmerston North 4410, New Zealand

6 Donvis Ltd., Palmerston North 4410, New Zealand 
end-product of their energy metabolism [2]. Several lineages of methanogenic archaea are core members of the microbiome of the ruminant foregut [3-5]. Of these, hydrogenotrophic methanogens are dominant in terms of both methane emissions and community composition [6, 7], with global surveys indicating that Methanobrevibacter gottschalkii and Methanobrevibacter ruminantium comprise $74 \%$ of the rumen methanogen community [5]. These organisms use molecular hydrogen $\left(\mathrm{H}_{2}\right)$ to reduce carbon dioxide $\left(\mathrm{CO}_{2}\right)$ to methane through the Wolfe cycle of methanogenesis [8, 9]. Rumen methanogens have also been identified that use formate, acetate, methyl compounds and ethanol as substrates, but usually do so in conjunction with $\mathrm{H}_{2}$ [5, 10-12]. Given their major contribution to greenhouse gas emissions, multiple programs are underway to mitigate ruminant methane production $[13,14]$. To date, most strategies have focused on direct inhibition of methanogens using chemical compounds or vaccines [15-18]. A promising alternative strategy is to modulate the supply of substrates to methanogens, such as $\mathrm{H}_{2}$, for example, through dietary or probiotic interventions $[14,19,20]$. To achieve this, while maintaining health and productivity of the host animal, requires an understanding of the processes that mediate substrate supply to methanogens within the rumen.

$\mathrm{H}_{2}$, the main substrate supporting ruminal methanogenesis, is primarily produced through fermentation processes [6]. Various carbohydrate fermentation pathways lead to the production of $\mathrm{H}_{2}$ as an end-product, together with volatile fatty acids and $\mathrm{CO}_{2}$ [21-23]. This process is supported by hydrogenases, which reoxidise cofactors reduced during carbohydrate fermentation and dispose of the derived electrons by producing $\mathrm{H}_{2}$. Although it is unclear which rumen microorganisms mediate $\mathrm{H}_{2}$ production in situ, a range of isolates have been shown to produce $\mathrm{H}_{2}$ in vitro [24-28]. For example, the model rumen bacterium $R$. albus 7 reoxidises the reduced ferredoxin and $\mathrm{NADH}$ formed during glucose fermentation by using two different $[\mathrm{FeFe}]$-hydrogenases depending on environmental conditions [29]. In addition, it is well-established that some rumen fungi and ciliates produce $\mathrm{H}_{2}$ via hydrogenosomes $[30,31]$. A debated source is the nitrogenase reaction, which produces $\mathrm{H}_{2}$ while fixing $\mathrm{N}_{2}$; whereas numerous rumen microorganisms encode putative nitrogenases [21], in situ data indicate that $\mathrm{N}_{2}$ fixation occurs at negligible rates in the rumen $[32,33]$. A large proportion of the $\mathrm{H}_{2}$ produced by hydrogenogenic fermenters is directly transferred to hydrogenotrophic methanogens, in an ecological process known as interspecies hydrogen transfer $[25,34]$. Particularly remarkable are the endosymbiotic and ectosymbiotic associations of methanogens, such as $M$. ruminantium, with rumen ciliates [35-37]. In addition to providing a continual substrate supply for methanogens, such symbioses benefit fermenters by maintaining $\mathrm{H}_{2}$ at sufficiently low concentrations for fermentation to remain thermodynamically favourable [38].

Various hydrogenotrophic bacteria are thought to compete with methanogens for the rumen $\mathrm{H}_{2}$ supply. Most attention has focused on acetogens, which mediate conversion of $\mathrm{H}_{2} / \mathrm{CO}_{2}$ to acetate using [FeFe]-hydrogenases [39]. Several genera of acetogens have been isolated from the rumen, including Eubacterium [40], Blautia [41] and Acetitomaculum [42]. However, molecular surveys indicate their abundance is generally lower than hydrogenotrophic methanogens [43-45]. This is thought to reflect that methanogens outcompete acetogens owing to the higher free energy yield of their metabolic processes, as well as their higher affinity for $\mathrm{H}_{2}$. The dissolved $\mathrm{H}_{2}$ concentration fluctuates in the rumen depending on diet, time of feeding and rumen turnover rates, but is generally at concentrations between 400 and $3400 \mathrm{~nm}$ [46]; these concentrations are typically always above the threshold concentrations required for methanogens $(<75 \mathrm{nM})$ but often below those of acetogens $(<700 \mathrm{~nm})$ [47]. Despite this, it has been proposed that stimulation of acetogens may be an effective strategy for methane mitigation in methanogen-inhibited scenarios [14, 20, 48, 49]. Various microorganisms have also been isolated from cows and sheep that support anaerobic hydrogenotrophic respiration, including dissimilatory sulfate reduction (e.g., Desulfovibrio desulfuricans) [50, 51], fumarate and nitrate reduction (e.g., Selenomonas ruminantium, Wolinella succinogenes) [52- 59] and trimethylamine $\mathrm{N}$-oxide reduction (e.g., Denitrobacterium detoxificans) [60]. The first described and most comprehensively studied of these hydrogen oxidisers is $W$. succinogenes, which mediates interspecies hydrogen transfer with $R$. albus [25]. In all cases, respiratory electron transfer via membrane-bound $[\mathrm{NiFe}]$-hydrogenases and terminal reductases generates a proton-motive force that supports oxidative phosphorylation [61]. It is generally assumed that these pathways are minor ones and are limited by the availability of oxidants. Promisingly, it has been observed that dietary supplementation with fumarate, sulfate, or nitrate can significantly reduce methane production in cattle, likely by stimulating alternative pathways of $\mathrm{H}_{2}$ consumption [62, 63].

We postulate that mitigating methane emissions, while maintaining animal productivity, depends on understanding and controlling $\mathrm{H}_{2}$ utilisation by methanogens. This requires a system-wide perspective of the schemes for production and concomitant utilisation of $\mathrm{H}_{2}$ in the rumen. To facilitate this, we determined which organisms and enzymes are primarily responsible for $\mathrm{H}_{2}$ production and consumption in rumen. First, we screened genome, metagenome and metatranscriptome data sets [21, 64, 65] to identify microbial genera, metabolic pathways and hydrogenase classes $[66,67]$ that mediate $\mathrm{H}_{2}$ metabolism. We demonstrate that ruminants harbour a diverse community of 
hydrogenogenic fermenters and hydrogenotrophic methanogens, acetogens and sulfate, fumarate and nitrate reducers. Second, we used the model system of the $\mathrm{H}_{2}$-producing carbohydrate fermenter $R$. albus 7 and the $\mathrm{H}_{2}$-utilising fumarate-reducing syntrophic partner Wolinella succinogenes DSM $1740[25,54,55,68]$ to gain a deeper mechanistic understanding of how and why ruminant bacteria regulate $\mathrm{H}_{2}$ metabolism. We observed significant differences in the growth, transcriptome and metabolite profiles of these bacteria in co-culture compared with pure culture. Finally, we compared gene expression profiles associated with $\mathrm{H}_{2}$ metabolism between low versus high methane yield sheep [64]. It was recently proposed, on the basis of community structure analysis, that fewer $\mathrm{H}_{2}$-producing bacteria inhabit low methane yield sheep [69]. In this work, we provide evidence for an alternative explanation: $\mathrm{H}_{2}$ uptake through non-methanogenic pathways accounts for these differences. Whereas the enzymes mediating fermentative $\mathrm{H}_{2}$ production are expressed at similar levels, those supporting $\mathrm{H}_{2}$ uptake through acetogenesis, fumarate reduction and nitrate ammonification pathways are highly upregulated in low methane yield sheep. In turn, these findings support that strategies to promote alternative $\mathrm{H}_{2}$ uptake pathways, including through dietary modulation, may significantly reduce methane emissions.

\section{Materials and methods}

\section{Comparative genomic analysis}

The protein sequences of the 501 genomes of cultured rumen bacteria (410 from Hungate Collection [21], 91 from other sources) were retrieved from the Joint Genome Institute (JGI) genome portal. These sequences were then screened against local protein databases for the catalytic subunits of the three classes of hydrogenases (NiFehydrogenases, FeFe-hydrogenases, Fe-hydrogenases), nitrogenases (NifH), methyl-CoM reductases (McrA), acetyl-CoA synthases (AcsB), adenylylsulfate reductases (AprA), dissimilatory sulfite reductases (DsrA), alternative sulfite reductases (AsrA), fumarate reductases (FrdA), dissimilatory nitrate reductases (NarG), periplasmic nitrate reductases (NapA), ammonia-forming nitrite reductases (NrfA), DMSO/TMAO reductases (DmsA) and cytochrome $b d$ oxidases (CydA). Hydrogenases were screened using the HydDB data set [66, 67], targeted searches were used to screen six protein families (AprA, AsrA, NarG, NapA, NrfA, DmsA, CydA) and comprehensive custom databases were constructed to screen five other protein families (NifH, McrA, AcsB, DsrA, FrdA) based on their total reported genetic diversity [70-74]. A custom Python script incorporating the Biopython package [75] was designed to produce and parse BLAST results (https://github.com/w oodlaur189/get_flanks_blast/releases). This script was used to batch-submit the protein sequences of the 501 downloaded genomes as queries for BLAST searches against the local databases. Specifically, hits were initially called for alignments with an e-value threshold of $1 \mathrm{e}-50$ and the resultant XML files were parsed. Alignments producing hits were further filtered for those with coverage values exceeding $90 \%$ and percent identity values of $30-70 \%$, depending on the target, and hits were subsequently manually curated. Table S1 and S2 provide the FASTA protein sequences, alignment details and distribution summaries of the filtered hits. For hydrogenases, the protein sequences flanking the hydrogenase large subunits were also retrieved; these sequences were used to classify group A [FeFe]-hydrogenases into subtypes (A1-A4), as previously described [66], and retrieve diaphorase sequences (HydB) associated with the A3 subtype. Partial [FeFe]hydrogenase protein sequences from six incompletely sequenced rumen ciliates and fungi genomes were retrieved through targeted blastP searches [76] in NCBI.

\section{Metagenomic and metatranscriptomic analysis}

We analysed previously published data sets of 20 paired metagenomes and metatranscriptomes of sheep rumen contents [64]. All profiles were derived from the rumen contents of age-matched, pelleted lucerne-fed rams that were collected $4 \mathrm{~h}$ after morning feeding and subject to paired-end sequencing on the HiSeq2000 platform [64]. The samples were taken from 10 rams at two different sampling dates based on their measured methane yields $[64,77]$; four rams were consistently low yield, four were consistently high yield, and two others switched in methane yield between the sampling dates (Table S3). The metagenome and metatranscriptome data sets analysed are accessible at the NCBI Sequence Read Archive (SRA; http://www.ncbi. nlm.nih.gov/sra) accession numbers SRA075938, and SRX1079958-SRX1079985 under bioproject number PRJNA202380. Each metagenome and metatranscriptome was subsampled to an equal depth of five million reads using seqtk (https://github.com/lh3/seqtk) seeded with parameter -s100. Subsampled data sets were then screened in DIAMOND (default settings, one maximum target sequence per query) [78] using the protein sequences retrieved from the 507 rumen microbial genomes $(\mathrm{NiFe}-$ hydrogenases, FeFe-hydrogenases, Fe-hydrogenases, HydB, NifH, McrA, AcsB, AprA, DsrA, AsrA, FrdA, NarG, NapA, NrfA, DmsA, CydA). Results were then filtered (length of amino acid $>40$ residues, sequence identity $>65 \%$ ). Subgroup classification and taxonomic assignment of the hydrogenase reads was based on their closest match 
to the hydrogenase data set derived from the 507 genomes at either 65 or $85 \%$ identity. The full pipeline used is provided on GitHub (https://github.com/Cecilia-Wang/Dia mond_line_reader_pipeline). The hits obtained are shown in Table S4 and S5. The number of reads with the rumenspecific hydrogenase data set $(15,464$ metagenome hits, 40,485 metatranscriptome hits) exceeded those obtained by screening with the generic data set from HydDB [67] (12,599 metagenome reads, 31,155 metatranscriptome reads), verifying that the rumen data set comprehensively captures hydrogenase diversity. For each data set, read count was normalised to account for the average length of each gene using the following formula: Normalised Read Count $=$ Actual Read Count $\times(1000 /$ average gene length $)$. Independent two-group Wilcoxon rank-sum tests were used to determine whether there were significant differences in the targets analysed between low and high methane yield sheep. Separate analyses were performed based on gene abundance, transcript abundance and RNA/DNA ratio.

\section{Bacterial growth conditions}

The bovine rumen isolates $R$. albus 7 [68] and Wolinella succinogenes DSM 1740 [54] were cultured anaerobically at $37^{\circ} \mathrm{C}$ in modified Balch medium [79] (Table S6). Precultures were grown in Balch tubes $(18 \times 150 \mathrm{~mm}$; Chemglass Life Sciences, Vineland, NJ) containing $20 \% \mathrm{v} / \mathrm{v}$ culture medium and sealed with butyl rubber stoppers crimped with aluminium caps. Cultures were grown in Pyrex side-arm flanks (Corning Inc., Corning, NY) containing $118 \mathrm{~mL}$ modified Balch medium. Two pre-cultures were grown before final inoculation, and all inoculum transfers were $5 \%(\mathrm{v} / \mathrm{v})$. The headspace consisted of $20 \%$ $\mathrm{CO}_{2}$ and $80 \% \mathrm{~N}_{2}$ for $R$. albus pure cultures and the cocultures, and $20 \% \mathrm{CO}_{2}$ and $80 \% \mathrm{H}_{2}$ for W. succinogenes pure cultures. Cultures were periodically sampled at $0,3,5$, 7, 9 and $11 \mathrm{~h}$ for metabolite analysis and bacterial quantification. Each culture was also sampled at 0 and $24 \mathrm{~h}$ to analyse $\mathrm{H}_{2}$ mixing ratios. Culture samples were immediately centrifuged $(16,000 \times g, 10 \mathrm{~min})$ in a bench-top centrifuge (Eppendorf, Hamburg, Germany). For metabolite analysis, the supernatant was collected and further centrifuged $(16,000 \times g, 10 \mathrm{~min})$ before high-performance liquid chromatography analysis.

\section{Bacterial quantification}

Quantitative PCR was used to quantify the copy numbers of $R$. albus and $W$. succinogenes under pure culture and co-culture. DNA was extracted from each pellet using the Fungal/Bacterial DNA MiniPrep kit according to the manufacturer's instructions (Zymo Research, Irvine, CA). Primers were designed to specifically amplify Rumal_2867 (R. albus glucokinase gene; FW: CTGGGATTCCTGAACTTTCC; RV: ATGCATACTGCGTTAG) and WS0498 (W. succinogenes flgL gene; FW: CAGACTATACCGATGCAACTAC; RV: GAGCGGAGGAGATCTTTAATC). For quantification, amplicons of each gene were cloned into pGEM-T Easy vectors (Promega, Madison, WI). Vector inserts were sequenced to confirm proper insertion. Standard curves extended from $10^{2}$ to $10^{10}$ copies $\mathrm{mL}^{-1}$. DNA was amplified with iTaq Universal SYBR Green (Bio-Rad) on a LightCycler 480 (Roche Holding AG, Basel, Switzerland). The amplification conditions were as follows: initial denaturation for 5 min at $95^{\circ} \mathrm{C}, 40$ amplification cycles $\left(15 \mathrm{~s}\right.$ at $95^{\circ} \mathrm{C}, 20 \mathrm{~s}$ at $60{ }^{\circ} \mathrm{C}, 10 \mathrm{~s}$ at $68^{\circ} \mathrm{C}$ ) and final extension for $5 \mathrm{~min}$ at $68^{\circ} \mathrm{C}$. Fluorescence was read during the extension phase of each cycle. The linear regression of the standard curve resulted in an $R^{2}>0.99$, efficiencies were between $97.8-98.5 \%$, and primers were confirmed to not cross-react. Two-tailed tests were done on sample data using SAS Studio (SAS Institute Inc., Cary, NC) to compare growth rates between single and co-cultures. All populations were normally and independently distributed with equal variances.

\section{Liquid and gas metabolite analysis}

The concentrations of acetate, ethanol, fumarate, succinate and formate in the culture supernatants were analysed using an Ultra-Fast Liquid Chromatograph (UFLC; Shimadzu, Kyoto, Japan). The UFLC consisted of a DGU-20A5 degasser, a SIL-20ACHT autosampler, an LC-20AT solvent delivery unit, an RID-10A refractive index detector, a CBM-20A system controller, and a CTO-20AC column oven. The mobile phase was $5 \mathrm{~mm} \mathrm{H}_{2} \mathrm{SO}_{4}$ passed through an Aminex HPX-87H ion exclusion column (Bio-Rad, Hercules, CA) at a flow rate of $0.4 \mathrm{~mL} \mathrm{~min}{ }^{-1}, 25^{\circ} \mathrm{C}$. Percentage mixing ratios of $\mathrm{H}_{2}$ were measured using a gas chromatograph (GC; Gow-Mac Series 580 Thermal Conductivity Gas Chromatograph, Gow-Mac Instrument Co., Bethlehem, PA). Samples were withdrawn directly from the culture tube in a gas-tight syringe and $0.5 \mathrm{~mL}$ was injected into $\mathrm{GC}$ for analysis using $\mathrm{N}_{2}$ as the carrier gas. The flow rate was 60 $\mathrm{mL} \mathrm{min}^{-1}$, the detector was set to $80^{\circ} \mathrm{C}$, the injector was set to $80^{\circ} \mathrm{C}$ and the oven was set to $75^{\circ} \mathrm{C}$. For both liquid and gas analyses, peak retention times and peak area were compared to standards of known concentration.

\section{RNA extraction and sequencing}

Each pure culture and co-culture used for transcriptome analysis was grown in duplicate in Balch tubes. Growth was monitored until the cultures were in mid-exponential phase; the change in $\mathrm{OD}_{600}$ at this phase was 0.14 for $W$. succinogenes, 0.20 for $R$. albus and 0.35 for the co-culture. At mid-exponential phase, $5 \mathrm{~mL}$ cultures were harvested by 
centrifugation $\left(13,000 \times g, 4{ }^{\circ} \mathrm{C}\right)$. Cell pellets were resuspended in $400 \mu \mathrm{L}$ fresh lysis buffer (5 mM EDTA, 0.5\% SDS, $25 \mathrm{~mm}$ lysozyme, $250 \mathrm{U} \mathrm{mL}^{-1}$ mutanolysin and 150 $\mu \mathrm{g} \mathrm{mL}^{-1}$ proteinase $\mathrm{K}$ in $25 \mathrm{~mm}$ sodium phosphate buffer, $\mathrm{pH}$ 7.0) and incubated under oxic conditions for $30 \mathrm{~min}$ at $55^{\circ} \mathrm{C}$ with periodic vortexing. RNA was subsequently extracted under oxic conditions using an RNeasy Mini Kit following the manufacturer's protocol, including all optional steps (Qiagen, Hilden, Germany), and eluted with $50 \mu \mathrm{L}$ ultra-pure DEPC-treated water (Invitrogen, Carlsbad, CA). RNA quantity, quality and integrity were confirmed by Qubit Fluorometry (Invitrogen, Carlsbad, CA), Nanodrop UV-Vis Spectrophotometry (Thermo Fisher Scientific, model 2300c), and agarose gel electrophoresis respectively. Bacterial rRNA was removed from $1 \mu \mathrm{g}$ of total RNA with the MicrobExpress Kit (Life Technologies, Carlsbad, CA). Libraries were prepared on the enriched mRNA fraction using the Stranded RNA Sample Prep Kit (Illumina, San Diego, CA). The barcoded libraries were pooled in equimolar concentration and sequenced on one lane for 101 cycles on a HiSeq2000 using a TruSeq SBS Sequencing Kit (Version 3). Fastq files were generated and demultiplexed with the bc12fastq Conversion Software (Illumina, version 1.8.4). The RNA-seq data were analysed using CLC Genomics Workbench version 5.5.1 (CLC Bio, Cambridge, MA). RNA-seq reads were mapped onto the reference genome sequences of $R$. albus 7 [80] and Wolinella succinogenes DSM 1740 [81] (Table S7 \& S8). The RNA-seq output files were analysed for statistical significance as described [82] and $q$ values were generated using the $q$ value package in $\mathrm{R}$ [83]. Predicted subsystems and functions were downloaded and aligned to the RNA-seq transcriptional data using the RAST Server [84].

\section{Results}

\section{$\mathrm{H}_{2}$ metabolism is a common and diverse trait among rumen bacteria, archaea and eukaryotes}

We searched the 501 reference genome sequences of rumen bacteria and archaea [21] for genes encoding the catalytic subunits of $\mathrm{H}_{2}$-consuming and $\mathrm{H}_{2}$-producing enzymes (Table S1 \& S2). Of these, 65\% encoded the capacity to metabolise $\mathrm{H}_{2}$ via [FeFe]-hydrogenases (42\%), [NiFe]-hydrogenases $(31 \%),[\mathrm{Fe}]$-hydrogenases $(2.4 \%)$ and/or nitrogenases $(23 \%)$. This suggests that $\mathrm{H}_{2}$ metabolism is a widespread trait among rumen microorganisms. We also identified multiple partial sequences of group A1 $[\mathrm{FeFe}]$-hydrogenases in the incomplete genomes of six rumen fungi and ciliates. This is consistent with the known ability of these microorganisms to produce $\mathrm{H}_{2}$ during cellulose fermentation [31]. The 329 hydrogenase- and nitrogenase-positive genomes spanned 108 genera, 26 orders, 18 classes and 11 phyla (Fig. 1; Figure S1; Table S1 \& S2).

We then classified the hydrogenases identified into subgroups. To do so, we used the phylogeny-based, functionally-predictive classification scheme of $\mathrm{HydDB}$ [67], which has been used to understand $\mathrm{H}_{2}$ metabolism in a range of organisms and ecosystems [85-89]. In total, 273 strains encoded hydrogenases from classes that primarily evolve $\mathrm{H}_{2}$ under physiological conditions (Table S2). These include group A1 and B [FeFe]hydrogenases and group $4 \mathrm{e}[\mathrm{NiFe}]-$ hydrogenases that couple ferredoxin oxidation to $\mathrm{H}_{2}$ production in anaerobic bacteria [90-92]. However, the most widespread hydrogenases are the group A3 [ $\mathrm{FeFe}]$-hydrogenases, which were encoded in 43 genera, among them well-characterised carbohydrate fermenters such as Ruminococcus, Lachnoclostridium and Bacteroides. These hydrogenases form heterotrimeric complexes, together with diaphorase subunits, that mediate the recently-discovered process of electron-confurcation: coupling co-oxidation of NADH and ferredoxin produced during fermentative carbon degradation to production of $\mathrm{H}_{2}[29,93]$. This reversible complex can also support hydrogenotrophic acetogenesis [94]. By retrieving the genes immediately upstream and downstream, we verified that the diaphorase subunits $(\mathrm{HydB})$ of this complex were co-encoded with the retrieved hydrogenase subunits (Fig. 1; Table S2).

In addition, multiple organisms encoded hydrogenases and terminal reductases known to support hydrogenotrophic growth (Fig. 1). All 21 methanogen genomes surveyed harboured [NiFe]-hydrogenases together with the signature gene of methanogenesis (mcrA) (Fig. 1; Table S2). These include 14 Methanobrevibacter strains, which encoded a complete set of enzymes for mediating hydrogenotrophic methanogenesis through the Wolfe cycle [8], including the [Fe]-hydrogenase and the groups $3 \mathrm{a}, 3 \mathrm{c}, 4 \mathrm{~h}$ and $4 \mathrm{i}[\mathrm{NiFe}]$-hydrogenases. Seven genomes encoded both $[\mathrm{FeFe}]$-hydrogenases (A2, A3) and the marker gene for acetogenesis (acsB) (Table S2), including known hydrogenotrophic acetogens Blautia schinkii [41] and Acetitomaculum ruminis [42]. Several subgroups of the group $1[\mathrm{NiFe}]$-hydrogenases, all membrane-bound enzymes known to support hydrogenotrophic respiration [66, 95], were also detected. Most notably, various Selenomonas, Mitsuokella and Wolinella strains encoded such hydrogenases together with the signature genes for fumarate reduction $(f r d A)$ and nitrate ammonification (narG, napA, nrfA). As anticipated, the group 1b $[\mathrm{NiFe}]$-hydrogenase and $d s r A$ gene characteristic of hydrogenotrophic sulfate reduction were also encoded in the three genomes of ruminal Desulfovibrio isolates (Fig. 1; Table S2). 


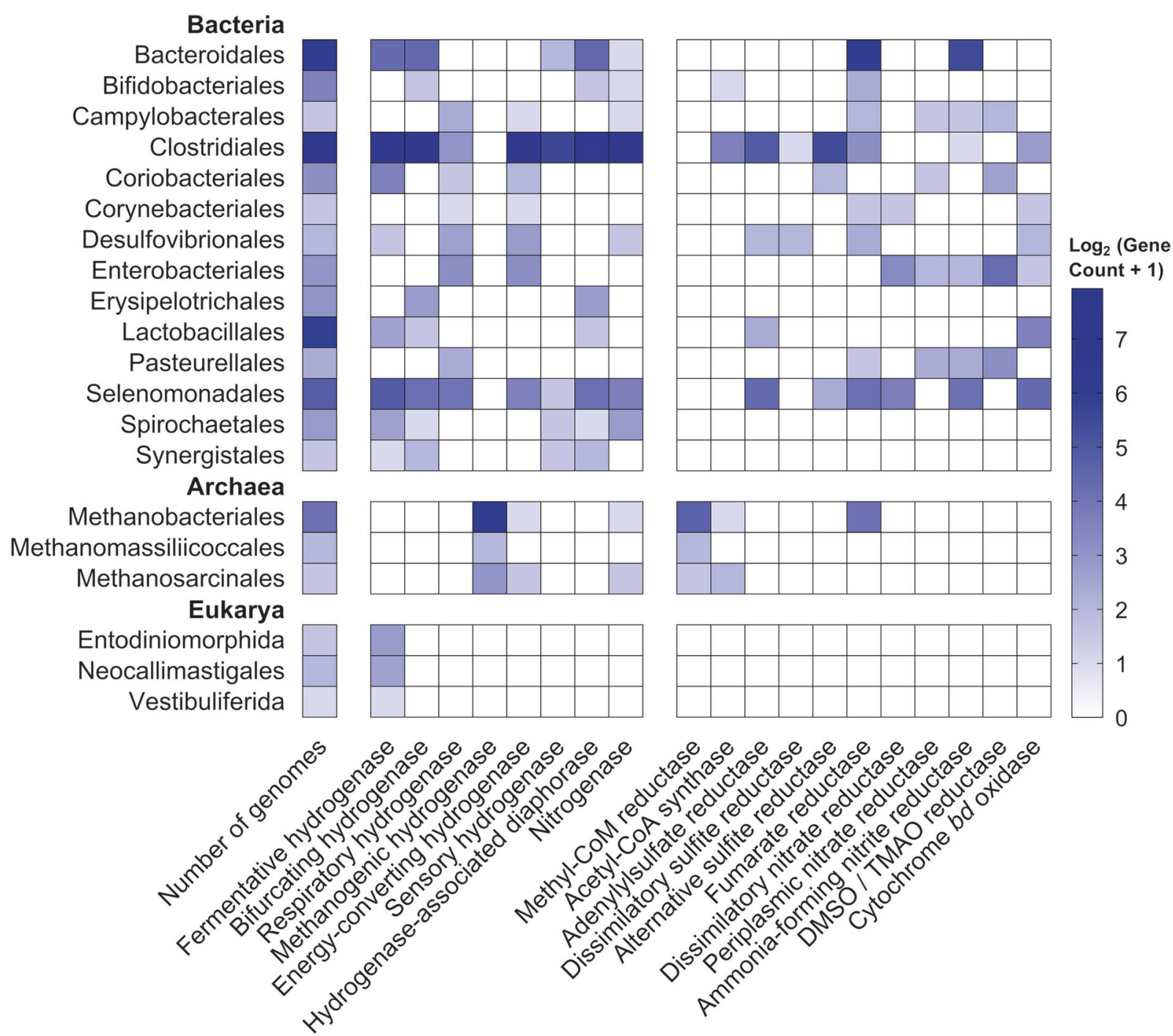

Fig. 1 Heatmap showing distribution of enzymes mediating $\mathrm{H}_{2}$ production and $\mathrm{H}_{2}$ consumption in orders of rumen microorganisms. Results are shown based on screens of the 501 genomes of cultured rumen bacteria and archaea (410 from the Hungate collection plus 91 other genomes). Partial hydrogenase sequences were also retrieved and classified from four rumen ciliates and two rumen fungi. The left-hand side of the heatmap shows the distribution of the catalytic subunits of enzymes that catalyse $\mathrm{H}_{2}$ oxidation and production. These are divided into fermentative hydrogenases $\left(\mathrm{H}_{2}\right.$-producing; group $\mathrm{A} 1, \mathrm{~A} 2$, B FeFehydrogenases), bifurcating hydrogenases (bidirectional; group A3, A4 FeFe-hydrogenases), respiratory hydrogenases $\left(\mathrm{H}_{2}\right.$-uptake; group $1 \mathrm{~b}$, 1c, 1d, 1f, 1i, 2d NiFe-hydrogenases), methanogenic hydrogenases $\left(\mathrm{H}_{2}\right.$-uptake; group $1 \mathrm{k}, 3 \mathrm{a}, 3 \mathrm{c}, 4 \mathrm{~h}, 4 \mathrm{i} \mathrm{NiFe}$-hydrogenases, Fe-hydrogenases), energy-converting hydrogenases (bidirectional; group $4 \mathrm{a}, 4 \mathrm{c}$, 4e, 4f, 4g NiFe-hydrogenases), sensory hydrogenases (group C FeFe-

\section{$\mathrm{H}_{2}$ is mainly produced in sheep by clostridial electron-bifurcating [FeFe]-hydrogenases and consumed by [NiFe]-hydrogenases of methanogens and selenomonads}

We then investigated the relative abundance and expression levels of the retrieved hydrogenases in rumen communities. hydrogenases) and nitrogenases $\left(\mathrm{H}_{2}\right.$-producing; $\left.\mathrm{NifH}\right)$. The right-hand side shows the distribution of the catalytic subunits of key reductases in $\mathrm{H}_{2}$ consumption pathways. They are genes for methanogenesis (McrA, methyl-CoM reductase), acetogenesis (AcsB, acetyl-CoA synthase), sulfate reduction (DsrA, dissimilatory sulfite reductase; AprA, adenylylsulfate reductase; AsrA, alternative sulfite reductase), fumarate reduction (FrdA, fumarate reductase), nitrate ammonification (NarG, dissimilatory nitrate reductase; NapA, periplasmic nitrate reductase; NrfA, ammonia-forming nitrite reductase), dimethyl sulfoxide and trimethylamine $N$-oxide reduction (DmsA, DMSO and TMAO reductase) and aerobic respiration (CydA, cytochrome $b d$ oxidase). Only hydrogenase-encoding orders are shown. Table S2 shows the distribution of these enzymes by genome, Figure S1 depicts hydrogenase subgroup distribution by class, and Table S1 lists the FASTA sequences of the retrieved reads

To do so, we used 20 pairs of metagenomes and metatranscriptomes that were previously sequenced from the rumen contents of age- and diet-matched farmed sheep [64] (Table S3). Screening these data sets with hydrogenases retrieved from the rumen microbial reference genomes yielded 15,464 metagenome hits $(0.015 \%$ of all reads) and 40,485 metatranscriptome hits $(0.040 \%)$ (Table S4). Across 
the metagenomes, the dominant hydrogenase reads originated from eleven subgroups (A1, A2, A3, B, 3a, 3c, 4e, 4g, 4h, 4i, Fe) (Fig. 2a \& S2a) and three taxonomic orders (Clostridiales, Methanobacteriales, Selenomonadales) (Fig. 2c \& S3a); this is concordant with the hydrogenase content in the genomes of the dominant community members [64, 65] (Table S2). Metatranscriptome analysis indicated these genes were differentially expressed: whereas A3, 1d, 3a, 3c and 4g genes were highly expressed (RNA/ DNA expression ratio $>4$ ), others were expressed at moderate (A1, A2, Fe; ratio 1.5-2.5) or low levels $(\mathrm{B}, 4 \mathrm{e}, 4 \mathrm{~h}, 4 \mathrm{i}$; ratio $<1.5$ ) (Figure S2 \& S3; Table $\mathrm{S} 1$ ). Though putative nitrogenase genes ( if $H$ ) were detected, expression ratios were low (av. 0.45), suggesting nitrogen fixation is not a significant $\mathrm{H}_{2}$ source in sheep (Figure $\mathrm{S} 4$ ). This is consistent with previous reports that nitrogenase activity in ovine rumen contents is low [33, 96].

Accounting for $54 \%$ of hydrogenase transcripts detected (Figs. 2b, 3a, S2), group A3 [FeFe]-hydrogenases appear to be the primary catalysts of $\mathrm{H}_{2}$ production in ruminants. We assigned the retrieved transcripts to taxa based on their closest hits to the rumen genome hydrogenase data set (Table S4). Clostridia accounted for the majority of the hits (Fig. 2d), including Ruminococcus (22\%), Saccharofermentans $(9.2 \%)$ and Lachnoclostridium (7.4\%) species known to fermentatively produce $\mathrm{H}_{2}$ [29, 34, 97] (Figure S5 \& S6). Transcripts from the characterised fermentative genera Bacteroides, Butyrivibrio, Clostridium and Sarcina were also moderately abundant. A further $21 \%$ of group A3 [FeFe]-hydrogenase hits were assigned to three a

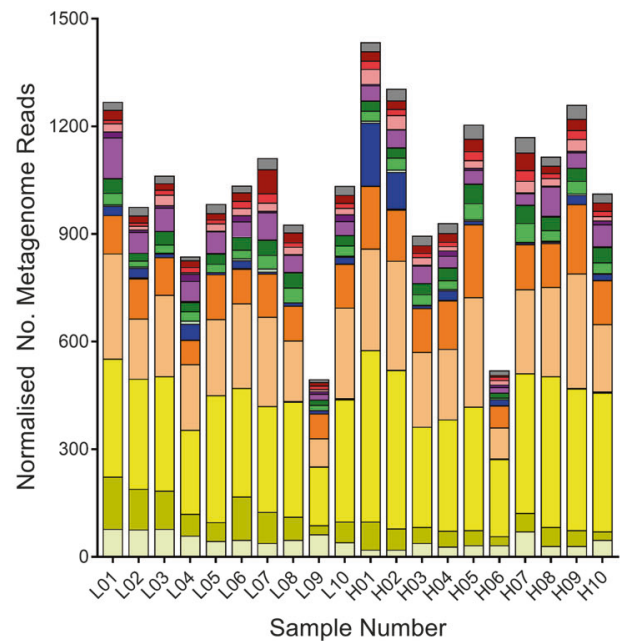

C

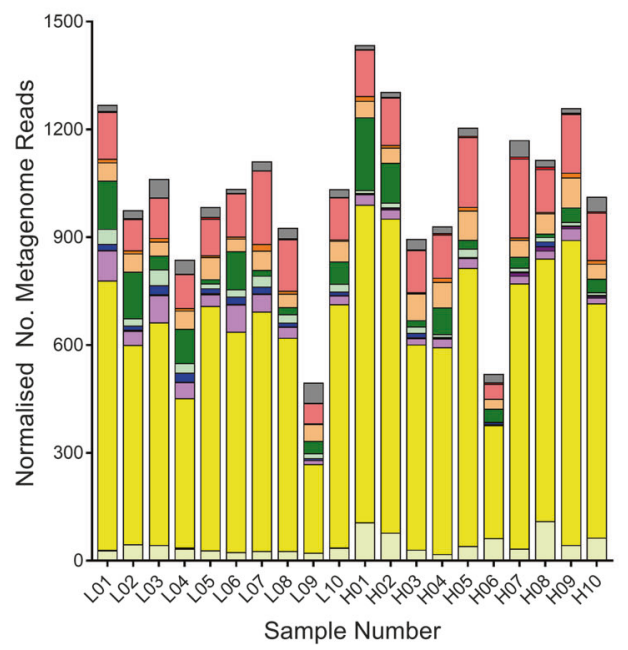

Fig. 2 Hydrogenase content in the metagenomes and metatranscriptomes of the microbial communities within rumen contents of high and low methane yield sheep. Hydrogenase content is shown based on hydrogenase subgroup $\mathbf{a}, \mathbf{b}$ and predicted taxonomic affiliation $\mathbf{c}, \mathbf{d}$ for metagenome data sets $\mathbf{a}, \mathbf{c}$ and metatranscriptome data sets $\mathbf{b}, \mathbf{d}$. Hydrogenase-encoding sequences were retrieved from 20 paired shotgun metagenomes and metatranscriptomes randomly b
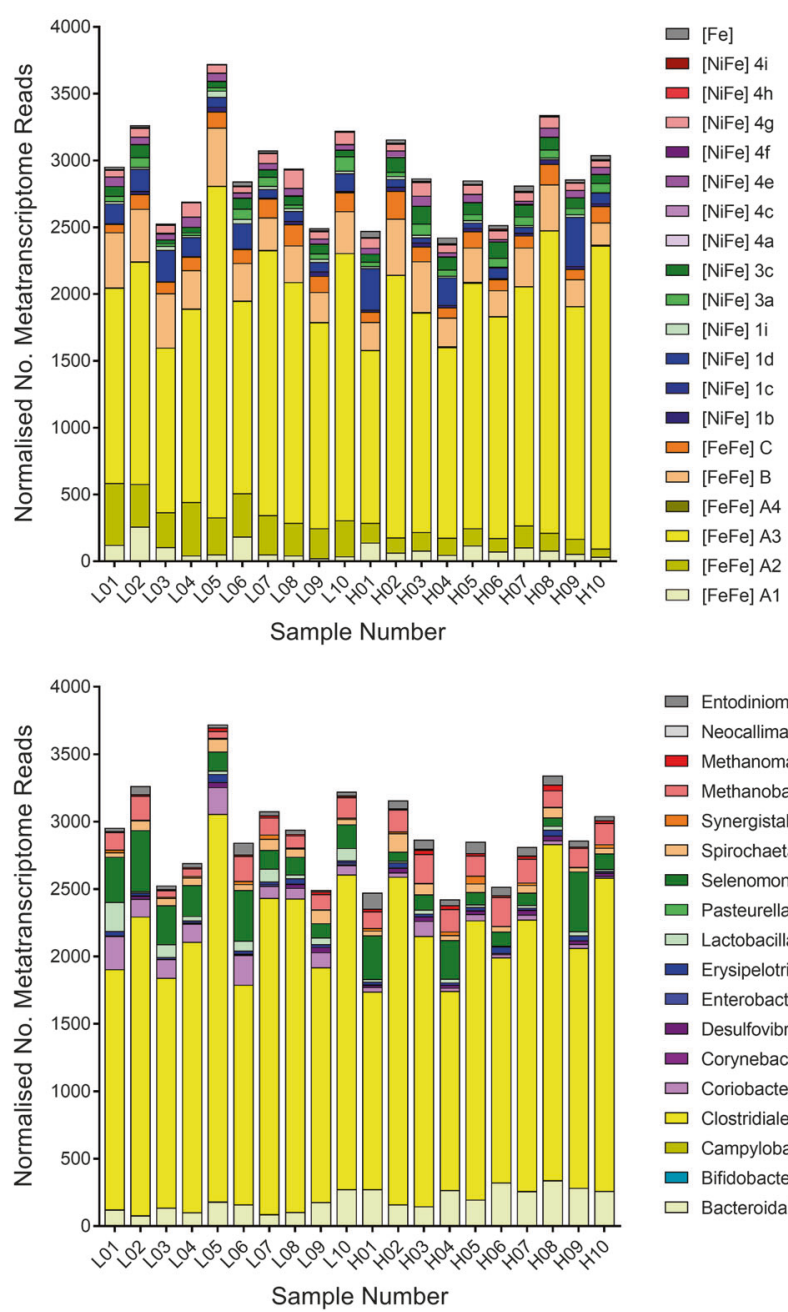
$\square$ Entodiniomorphida $\square$ Neocallimastigales
- Methanomassilicoccales
$\square$ Methanobacteriales
$\square$ Synergistales
$\square$ Spirochaetales
- Selenomonadales
$\square$ Pasteurellales
$\square$ Lactobacillales
- Erysipelotrichales
- Enterobacteriales
- Desulfovibrionales
- Corynebacteriales
$\square$ Coriobacteriales
$\square$ Clostridiales
$\square$ Campylobacterales
$\square$ Bifidobacteriales
$\square$ Bacteroidales

subsampled at five million reads. Reads were classified into hydrogenase subgroups and taxonomically assigned at the order level based on their closest match to the hydrogenases within the genomes screened (Fig. 1). L01 to L10 are data sets for sheep that were low methane yield at time of sampling, H01 to H10 are data sets from sheep that were high methane yield at time of sampling (see Table S3 for full details) 

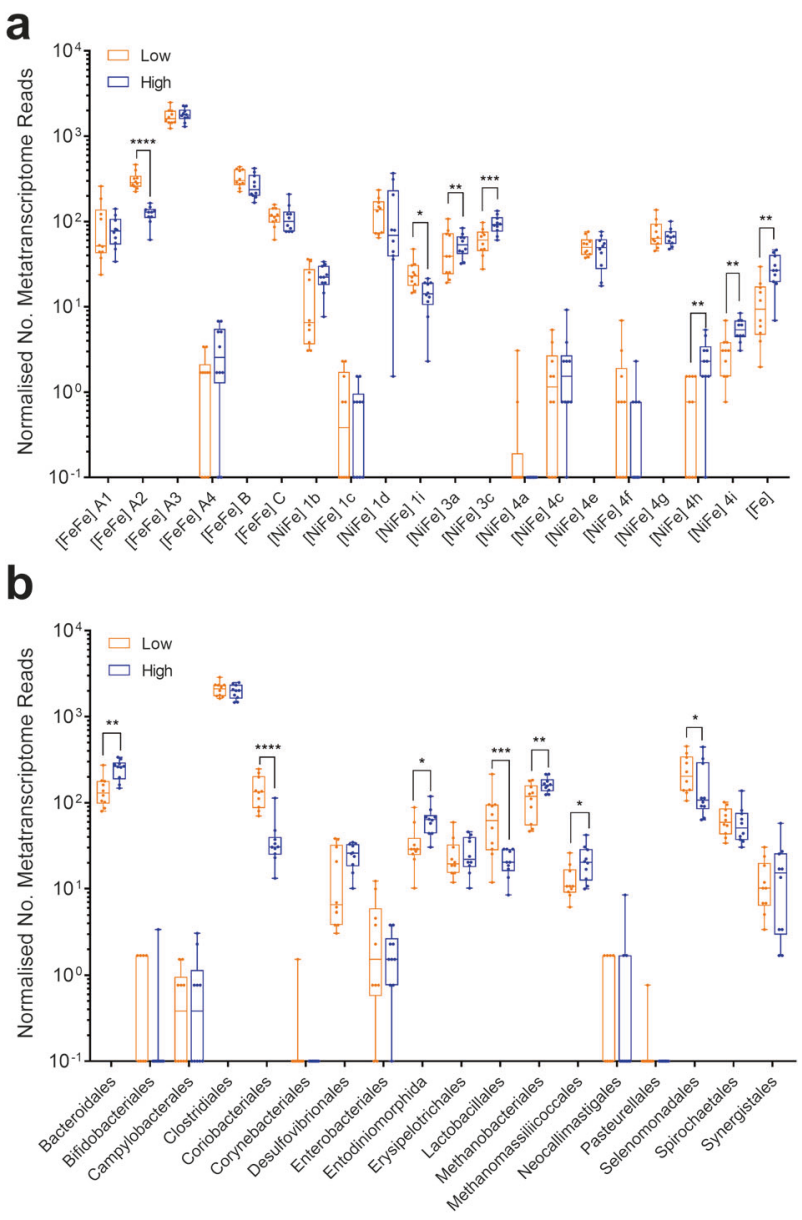

C

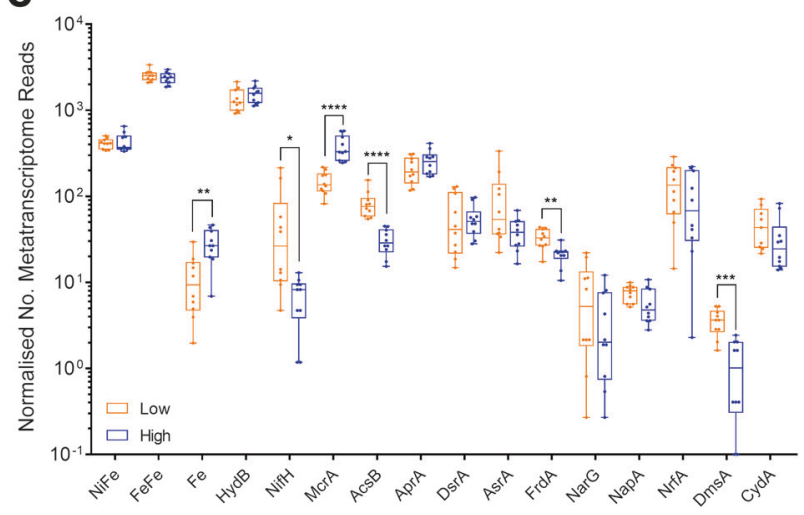

uncharacterised cultured lineages within the Clostridia: Clostridiales R-7, Ruminococcaceae P7 and Lachnospiraceae YSB2008 (Figure S5 \& S6). This is compatible with our previous studies showing unclassified microorganisms, especially from R-7 group, are abundant in rumen [21]. $\mathrm{H}_{2}$-evolving hydrogenases from the A1 and B subgroups were also detected, but their RNA/DNA expression ratios were threefold lower than the A3 hydrogenases. Rumen ciliates such as Epidinium dominated A1 reads (Fig. 2d \& S5), but it is likely that their abundance in the data sets is underestimated owing to the minimal genome coverage of
Fig. 3 Comparison of expression levels of $\mathrm{H}_{2}$ production and $\mathrm{H}_{2}$ uptake pathways in low and high methane yield sheep. Results are shown for 10 metatranscriptome data sets each from low methane yield sheep (orange) and high methane yield sheep (blue) that were randomly subsampled at five million reads. a Normalised count of hydrogenase transcript reads based on hydrogenase subgroup. b Normalised count of hydrogenase transcript reads based on predicted taxonomic affiliation. c Normalised count of transcript reads of key enzymes involved in $\mathrm{H}_{2}$ production and $\mathrm{H}_{2}$ consumption, namely the catalytic subunits of [NiFe]-hydrogenases (NiFe), [FeFe]-hydrogenases $(\mathrm{FeFe}),[\mathrm{Fe}]-$ hydrogenases $(\mathrm{Fe})$, hydrogenase-associated diaphorases (HydB), nitrogenases (NifH), methyl-CoM reductases (McrA), acetyl-CoA synthases (AcsB), adenylylsulfate reductases (AprA), dissimilatory sulphite reductases (DsrA), alternative sulfite reductases (AsrA), fumarate reductases (FrdA), dissimilatory nitrate reductases (NarG), periplasmic nitrate reductases (NapA), ammoniaforming nitrite reductases (NrfA), DMSO/TMAO reductases (DmsA) and cytochrome $b d$ oxidases (CydA) are provided. For FrdA, NrfA and $\mathrm{CydA}$, the numerous reads from non-hydrogenotrophic organisms (e.g., Bacteroidetes) were excluded. Each boxplot shows the 10 datapoints and their range, mean and quartiles. Significance was tested using independent two-group Wilcoxon rank-sum tests ${ }^{*} p<0.05$; $* * p<0.01 ; * * * p<0.001 ; * * * * p<0.0001$; full $p$ values in Table S9, $\mathrm{S} 10$ and S11). Note the metagenome abundance and RNA/DNA ratio of these genes is shown in Figure S2 (hydrogenase subgroup), Figure S3 (hydrogenase taxonomic affiliation) and Figure $\mathrm{S} 4\left(\mathrm{H}_{2}\right.$ uptake pathways). A full list of metagenome and metatranscriptome hits is provided for hydrogenases in Table $\mathrm{S} 4$ and $\mathrm{H}_{2}$ uptake pathways in Table S5

these organisms to date. The expression of formate hydrogenlyases and other formate-dependent hydrogenases were extremely low in metatranscriptome data sets (Fig. 3c), indicating formate-dependent $\mathrm{H}_{2}$ production is not a major pathway in situ.

The metatranscriptome data sets indicate that multiple $\mathrm{H}_{2}$ uptake pathways operate in ruminants (Figs. 2 and 3). In agreement with historical paradigms [6], hydrogenotrophic methanogenesis appears to be the largest sink of $\mathrm{H}_{2}$; methanogens accounted for $5.3 \%$ of normalised hydrogenase reads (Fig. 2d) and methyl-CoM reductase ( $m c r A)$ is the most expressed of the reductases surveyed (Fig. 3c). Consistent with their central roles in the $\mathrm{CO}_{2}$-reducing pathway of methanogenesis [9], the $\mathrm{F}_{420}$-reducing [NiFe]-hydrogenase (3a) [98] and the heterodisulfide reductase-associated [NiFe]hydrogenase (3c) [99] of Methanobrevibacter species were among the most transcribed of all $\mathrm{H}_{2}$ uptake enzymes (Fig. 3a and S5). In contrast, the Eha-type (4h), Ehb-type (4i) and [Fe]-hydrogenases were expressed at lower levels (Fig. 3a and S5), reflecting their secondary roles in the physiology of methanogens [100-102]. There was also strong evidence that hydrogenotrophic acetogenesis may be a more significant ruminal $\mathrm{H}_{2}$ sink than previously recognised. Across the data set, acetyl-CoA synthases ( $a c s B ; 1135$ normalised reads) were expressed at a quarter of the level of methyl-CoM reductases ( $m c r A ; 5246$ normalised reads) (Fig. 3c). For $74 \%$ of the reads, the closest matches were to predicted hydrogenotrophic acetogens isolated from rumen, including 
Blautia, Acetitomaculum and Oxobacter (Figure S7 \& Table S5). Consistently, genes encoding close homologues of the group A2 and group A3 [FeFe]-hydrogenases from these genera were moderately expressed in the metatranscriptomes (3.7\%) (Figure S5). The other $a c s B$ reads likely originate from acetogens that use other electron donors, such as formate. No $a c s B$ hits from methanogens were detected.

Surprisingly, however, the most highly expressed $\mathrm{H}_{2}$ uptake hydrogenase overall is the group $1 \mathrm{~d}$ [NiFe]-hydrogenase of Selenomonadales (4.1\%) (Figs. 3a, b and S5). This enzyme is likely to mediate the long-known capacity of Selenomonas species to grow by hydrogenotrophic fumarate reduction and nitrate ammonification $[52,53,58]$. Consistently, fumarate reductases $(f r d A)$, nitrate reductases (narG) and ammonia-forming nitrite reductases (nrfA) homologous to those in $S$. ruminantium were expressed in the metatranscriptomes (Fig. 3c). Normalised nrfA expression was fivefold higher than $\operatorname{narG}$, indicating selenomonads may preferentially use external nitrite, although further studies are required to determine the source of nitrite, this compound is known to accumulate in the rumen depending on nitrate content of feed [103]. Reads corresponding to the group $1 \mathrm{~b}$ [NiFe]-hydrogenase, periplasmic nitrate reductase (napA), nrfA and frdA from Wolinella was also detected, but at low levels (Table S4 \& S5; Figure S5). Several other pathways in low abundance in the metagenome were also highly expressed, notably group $1 \mathrm{~b}[\mathrm{NiFe}]$-hydrogenases and $d s r A$ genes from Desulfovibrio species, as well as group 1i [NiFe]-hydrogenases from metabolically flexible Coriobacteriia (e.g., Slackia, Denitrobacterium) (Figure S4 \& S5). The expression levels of the group $1 \mathrm{~b}$ and $1 \mathrm{~d}$ [NiFe]hydrogenases, together with the functionally unresolved group $4 \mathrm{~g}$ [NiFe]-hydrogenases, were the highest of all hydrogenases in data sets (RNA/DNA ratio $>10$ ) (Figure S3). Though these findings need to be validated by activity-based studies in situ, they suggest that respiratory hydrogenotrophs are highly active and quantitatively significant $\mathrm{H}_{2}$ sinks in the rumen despite often being detected in low abundance [5].

\section{Culture-based studies demonstrate that hydrogenases mediating $\mathrm{H}_{2}$ production and uptake are differentially regulated in response to hydrogen levels}

In order to better understand how rumen bacteria regulate $\mathrm{H}_{2}$ metabolism, we performed a culture-based study using R. albus 7 and Wolinella succinogenes DSM 1740. This system was chosen given it is the best-studied example of rumen microorganisms that participate in interspecies hydrogen transfer [25] and is relevant to $\mathrm{H}_{2}$ transactions in the rumen, given Ruminococcus electron-bifurcating hydrogenases are the most abundant in the sheep metatranscriptome data sets (Figure S5). We compared the growth, transcriptome and extracellular metabolite profiles of these strains in either pure culture or co-culture when grown on modified fumarate-supplemented Balch medium (Table S6). The concentrations of the metabolites consumed and produced by the strains varied between the conditions (Table S1; Figure S8) in a manner consistent with previous reports $[24,25,29,54]$ and the transcriptomic results (Fig. 4). Based on the ratios of the products observed, $R$. albus fermentatively degraded cellobiose to $\mathrm{H}_{2}$, acetate and ethanol in pure culture (glucose $+3.3 \mathrm{ADP}+3.3 \mathrm{P}_{\mathrm{i}} \rightarrow 2.6$ $\mathrm{H}_{2}+1.3$ acetate +0.7 ethanol $+2 \mathrm{CO}_{2}+3.3$ ATP [29]) and $\mathrm{H}_{2}$ and acetate in co-culture (glucose $+4 \mathrm{ADP}+$ $4 \mathrm{P}_{\mathrm{i}} \rightarrow 4 \mathrm{H}_{2}+2$ acetate $+2 \mathrm{CO}_{2}+4$ ATP [29]) (Fig. 4a-c). $W$. succinogenes grew by hydrogenotrophic fumarate respiration under both conditions by using exogenously supplied $\mathrm{H}_{2}$ in pure culture and syntrophically-produced $\mathrm{H}_{2}$ in co-culture (Fig. 4-f). Hence, R. albus channels fermentation through the pathway that yields stoichiometrically more ATP, $\mathrm{H}_{2}$ and acetate, provided that $\mathrm{H}_{2}$ concentrations are kept sufficiently low through interspecies hydrogen transfer for this to be thermodynamically favourable (see Zheng et al. [29] for stoichiometric and thermodynamic considerations).

Transcriptome profiling revealed that $R$. albus tightly regulates the expression of its three hydrogenases (Fig. 4a, b). Overall, 133 genes were differentially expressed (fold change $>2, q$ value $<0.05$ ) in co-culture compared with pure culture (Table S7). Of these, the greatest fold change was the 111-fold downregulation of a putative eight-gene cluster encoding the ferredoxin-only hydrogenase (group A1 [FeFe]-hydrogenase), a bifunctional alcohol and aldehyde dehydrogenase, and regulatory elements including a putative sensory hydrogenase (group $\mathrm{C}$ [FeFe]hydrogenase) (Fig. 4a, b). By suppressing expression of these enzymes, $R$. albus can divert carbon flux from ethanol production to the more energetically efficient pathway of acetate production; acetate fermentation produces equimolar levels of NADH and reduced ferredoxin, which can be simultaneously reoxidised by the electron-bifurcating hydrogenase (group A3 [FeFe]-hydrogenase) (Fig. 4c). Glycolysis enzymes and the phosphate acetyltransferase, acetate kinase and electron-bifurcating hydrogenase of the acetate production pathway were expressed at similarly high levels under both conditions (Fig. 4a, b). However, there was a significant increase in the biosynthesis of thiamine pyrophosphate, a cofactor for pyruvate dehydrogenase complex, in co-culture (Fig. 4a).

The fermentation stoichiometries of R. albus 7 measured in pure culture compared with co-culture (Table 1) were the same as we previously reported for the bacterium at high vs low concentrations of $\mathrm{H}_{2}$ [29]. This suggests that the differences in regulation are primarily determined by $\mathrm{H}_{2}$ 


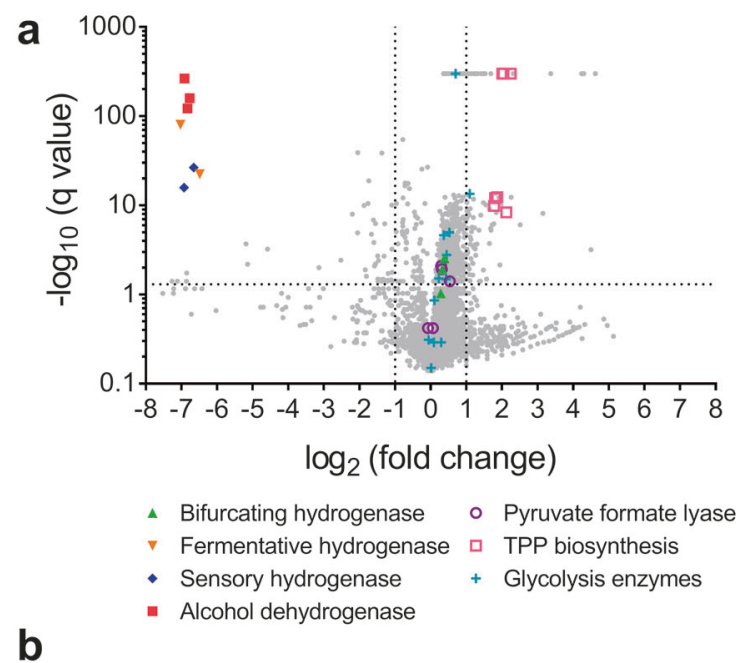

111 × downregulated: Group A1 (fermentative) and Group C (sensory) [FeFe]-hydrogenases

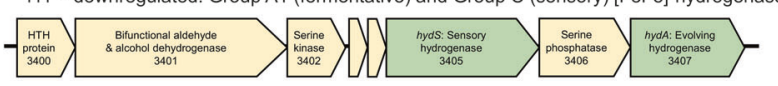

$1.26 \times$ upregulated: Group A3 (electron-bifurcating) [FeFe]-hydrogenase
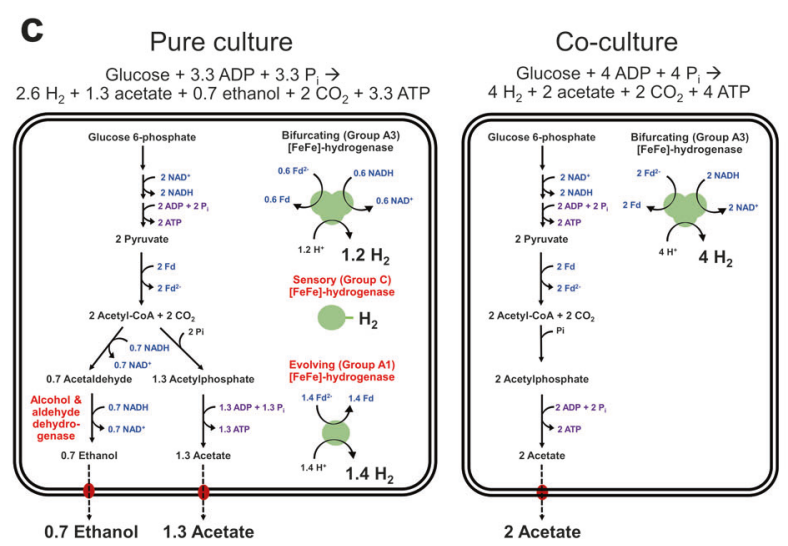

Fig. 4 Comparison of whole genome expression levels of Ruminococcus albus and Wolinella succinogenes in pure culture and co-culture. Pure cultures and co-cultures of Ruminococcus albus 7 a-c and Wolinella succinogenes DSM $1740 \mathbf{d}-\mathbf{f}$ were harvested in duplicate during midexponential phase and subject to RNA sequencing. a, d Volcano plots of the ratio of normalised average transcript abundance for co-cultures over pure cultures. Each gene is represented by a grey dot and key metabolic genes, including hydrogenases, are highlighted as per the legend. The horizontal dotted lines indicate $q$ values of 0.05 and the vertical dotted lines indicate twofold changes. b, d Predicted operon structure of the

levels, rather than by direct interactions with syntrophic partners. This regulation may be achieved through direct sensing of $\mathrm{H}_{2}$ by the putative sensory group $\mathrm{C}$ [FeFe]hydrogenase co-transcribed with the ferredoxin-only hydrogenase and alcohol dehydrogenase (Fig. 4e). In common with other enzymes of this class [66, 104, 105], this enzyme contains a subunit containing a $\mathrm{H}$-cluster for $\mathrm{H}_{2}$ binding and a PAS domain for signal transfer, as well as a

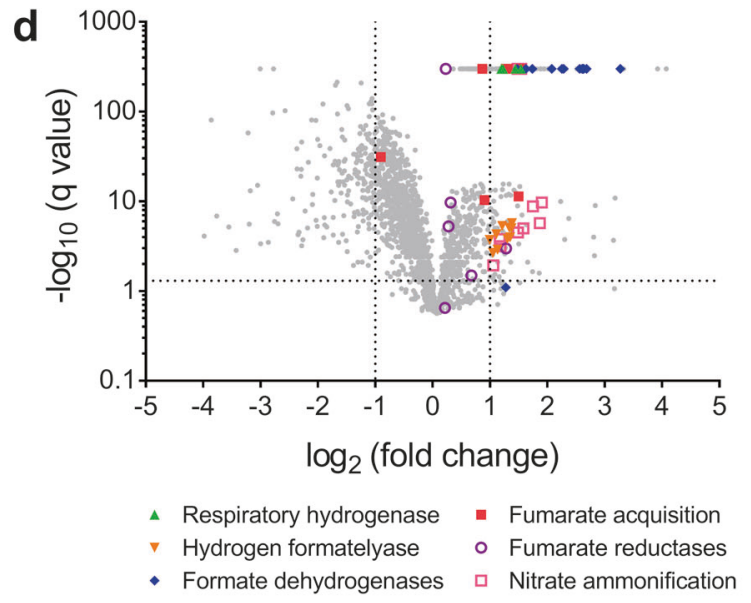

e

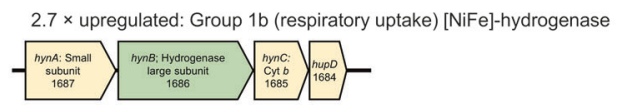

$2.4 \times$ upregulated: Group 4a (formate hydrogenlyase) [NiFe]-hydrogenases

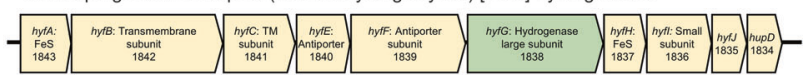

f

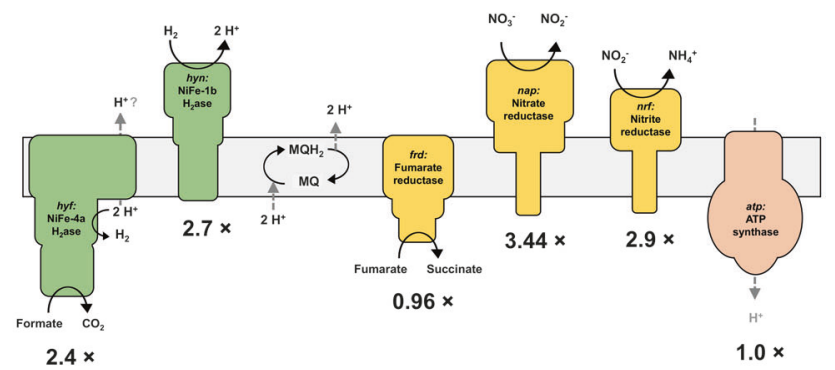

three hydrogenases of $R$. albus and two hydrogenases of W. succinogenes. e Comparison of dominant fermentation pathways of $R$. albus in pure culture (left) and co-culture (right) based on transcriptome reads and metabolite profiling. Three enzymes with decreased expression in coculture are in red font. f Respiratory chain composition of W. succinogenes in pure culture and co-culture based on transcriptome reads. Metabolite profiling indicated that the respiratory hydrogenase and fumarate reductases were active in both conditions. Proton translocation is thought to occur primarily through redox-loop mechanisms. A full list of read counts and expression ratios for each gene is provided in Table 1

putative serine or threonine phosphatase subunit that may modify downstream regulators. Thus, analogous to the wellstudied regulatory hydrogenases of aerobic bacteria [106, 107], this enzyme may directly sense $\mathrm{H}_{2}$ levels and induce expression of the alcohol/aldehyde dehydrogenase and ferredoxin-only hydrogenase when $\mathrm{H}_{2}$ concentrations are high through a feedback loop. $\mathrm{H}_{2}$ sensing may be a general mechanism regulating hydrogenase expression in 
Table 1 Comparison of growth parameters and metabolite profiles of Ruminococcus albus and Wolinella succinogenes in pure culture and co-culture

\begin{tabular}{llll}
\hline & $\begin{array}{l}\text { Ruminococcus } \\
\text { albus }\end{array}$ & $\begin{array}{l}\text { Wolinella } \\
\text { succinogenes }\end{array}$ & Co-culture \\
\hline $\begin{array}{l}\text { Growth parameters } \\
\text { Growth yield }\end{array}$ & $0.79 \pm 0.01$ & $0.36 \pm 0.01$ & $0.93 \pm 0.01$ \\
$\left(\mathrm{OD}_{600}\right)$ & & & \\
Specific growth & $0.58 \pm 0.19$ & $0.33 \pm 0.06$ & $0.57 \pm 0.34$ \\
rate $\left(\mathrm{h}^{-1}\right)$ & & & $(\mathrm{Ra})$ \\
& & & $0.54 \pm 0.11$ \\
& & & $(\mathrm{Ws})$ \\
Concentration changes of extracellular & metabolites & \\
Hydrogen $(\%)$ & +5.3 & -78.4 & $\mathrm{BDL}$ \\
Fumarate $(\mathrm{mM})$ & -5.5 & -46.3 & -43.1 \\
Succinate $(\mathrm{mM})$ & +2.2 & +54.6 & +55.4 \\
Acetate $(\mathrm{mM})$ & +21.8 & 0 & +32.4 \\
Ethanol $(\mathrm{mM})$ & +8.7 & 0 & +0.3 \\
Formate $(\mathrm{mM})$ & $\mathrm{BDL}$ & $\mathrm{BDL}$ & $\mathrm{BDL}$ \\
pH & -0.4 & -0.4 & -0.6 \\
\hline
\end{tabular}

Growth of pure cultures and co-cultures of Ruminococcus albus 7 and Wolinella succinogenes DSM 1740 was monitored by qPCR. Values show means \pm standard deviations of three biological replicates. Also shown is the change in extracellular $\mathrm{pH}$, percentage hydrogen gas (measured by gas chromatography), and concentrations of fumarate, succinate, acetate, ethanol and formate (measured by ultra-fast liquid chromatography) between 0 and $12 \mathrm{~h}$. Growth media was the same between the three conditions, except $80 \% \mathrm{H}_{2}$ was added for $W$. succinogenes growth, whereas no $\mathrm{H}_{2}$ was added for the other conditions. Full liquid metabolite measurements are shown in Figure S8. BDL = below detection limit ( $1 \mathrm{~mm}$ for formate, $0.1 \%$ for $\mathrm{H}_{2}$ ).

ruminants, given group $\mathrm{C}[\mathrm{FeFe}]$-hydrogenases are abundant in ruminant genome (Fig. 1), metagenome (Fig. 2a), and metatranscriptome data sets (Fig. $2 \mathrm{~b}$ and $3 \mathrm{a}$ ).

The transcriptome results also clarified understanding of hydrogenotrophic fumarate respiration by W. succinogenes (Fig. 4d). In both pure culture and co-culture, the group $1 \mathrm{~b}[\mathrm{NiFe}]$-hydrogenase, fumarate reductase and $\mathrm{F}_{1} \mathrm{~F}_{\mathrm{o}}$-ATPase that mediate this process were expressed at high levels (Table S7; Fig. 4f). A periplasmic asparaginase, aspartate ammonia-lyase and dicarboxylatebinding proteins were also highly expressed; this suggests that the organism can efficiently produce and import additional fumarate from amino acid sources (Table S7). In total, 352 genes were significantly differentially regulated in co-culture (fold change $>2, q$ value $<$ $0.05)$. The respiratory hydrogenase was among the upregulated genes (Fig. 4e), which may reflect the strain's faster growth rate in co-culture (Table 1). The periplasmic nitrate reductase and ammonia-forming nitrite reductase (Fig. 4f) were also induced, indicating some plasticity in electron acceptor usage, in line with the metatranscriptomic findings. Two formate dehydrogenases and a formate hydrogenlyase (group $4 \mathrm{a}[\mathrm{NiFe}]$-hydrogenase) were highly expressed in co-culture (Fig. 4d, e), indicating the bacterium can potentially use formate transferred from $R$. albus as a supplemental electron donor to $\mathrm{H}_{2}$ (Fig. 4a); consistently, R. albus is known to produce through formate pyruvate lyase activity and this enzyme was highly expressed in both pure culture and co-culture (Table S7), though any formate produced was below detection limits (1 mM) (Table 1$)$.

\section{Hydrogenotrophic acetogenesis, fumarate reduction and nitrate reduction pathways are significantly upregulated in low methane yield sheep}

Finally, we tested whether the abundance and expression of hydrogenases and $\mathrm{H}_{2}$ uptake pathways differed between high and low methane yield sheep. A current leading hypothesis, proposed on the basis of community composition [69], asserts that $\mathrm{H}_{2}$ production levels account for differences in methane yield between sheep. To the contrary, the expression levels of the dominant $\mathrm{H}_{2}$-evolving hydrogenases (e.g., group A3 [FeFe]-hydrogenases) and taxonomic orders (e.g., Clostridiales) were in fact extremely similar between the groups (Fig. 3a, b; Table S9 \& S10).

We therefore tested an alternative hypothesis: $\mathrm{H}_{2}$ utilisation through non-methanogenic pathways can reduce methane yield. In line with this hypothesis, the expression levels of the five methanogen hydrogenases and methyl$\mathrm{CoM}$ reductase are significantly reduced in low methane yield sheep (Fig. 3a, c; Table S9 \& S11), confirming a strong correlation with methane yield (Table S3). Concurrent increases in the gene expression for two major alternative $\mathrm{H}_{2}$ sinks were detected, namely acetogenesis $(a c s B ; p<0.0001)$ and fumarate reduction $(f r d A ; p=0.002)$ (Fig. 3c; Table S10), concomitant with significant increases in the expression levels of Blautia and Selenomonas hydrogenases (Figure S5). Expression levels of $n r f A$ were also on average 1.8-fold higher in low methane yield sheep, though there was much inter-sample variation in the read count for this gene. Whereas there are more transcripts of mcrA than other terminal reductases combined in high methane yield sheep, the transcript levels of $a c s B$ and $n r f A$ together exceed those of $m c r A$ in low methane yield sheep. Depending to what extent expression levels predict activity, hydrogenotrophic acetogens and selenomonads may therefore be more active than methanogens in low methane yield sheep and may significantly limit substrate supply for methanogenesis. Two other potential $\mathrm{H}_{2}$ sinks are also upregulated in the low methane yield sheep: the putative group $1 \mathrm{i}[\mathrm{NiFe}]$-hydrogenase of Coriobacteriia and, consistent with previous observations [65], the functionally unresolved group A2 [FeFe]-hydrogenase of Sharpea, Olsenella and Oribacterium (Fig. 3a, b, S5). 


\section{Discussion}

To summarise, $\mathrm{H}_{2}$ metabolism is a more widespread and complex process in ruminants than previously realised. Together, the genomic, metagenomic and metatranscriptomic surveys suggest that multiple orders of bacteria, archaea and eukaryotes encode and express enzymes mediating $\mathrm{H}_{2}$ production and consumption in the rumen. We infer that fermentative Clostridia are the main source of $\mathrm{H}_{2}$ in the rumen, which largely agrees with findings from activity-based and culture-based studies [6, 25, 26, 29], and that a range of methanogenic, acetogenic and respiratory hydrogenotrophs are present. Although this manuscript provides a relatively comprehensive understanding of $\mathrm{H}_{2}$ transactions in the rumen, it is probable that other uncharacterised organisms and genes also contribute to this process. A more-detailed perspective of which taxa mediate $\mathrm{H}_{2}$ cycling in the rumen would be possible by profiling the thousands of metagenome-assembled genomes recently recovered from ruminants [23, 108] and also better accounting for the role of the largely unsequenced rumen ciliates and fungi, which to date are underrepresented in genomic data sets. Further studies are also necessary to extend findings to other ruminants, such as bovines, and investigate how $\mathrm{H}_{2}$ cycling responds to environmental factors such as diet.

One of the most-important findings of this work is that the recently characterised electron-bifurcating hydrogenases appear to primarily mediate ruminal $\mathrm{H}_{2}$ production. These enzymes are highly upregulated compared with ferredoxinonly hydrogenases in situ and constitute over half of hydrogenase reads in these metatranscriptomes. We provide a rationale for this finding by showing that $R$. albus, a dominant $\mathrm{H}_{2}$ producer within the rumen, expresses its electron-bifurcating hydrogenase and suppresses its ferredoxin-only hydrogenase when grown syntrophically with Wolinella succinogenes. In this condition, $\mathrm{H}_{2}$ concentrations remain sufficiently low that the fermentation pathway producing higher levels of ATP, $\mathrm{H}_{2}$ and acetate remains thermodynamically favourable. In the rumen, where tight coupling of hydrogenogenic and hydrogenotrophic processes usually keeps $\mathrm{H}_{2}$ at sub-micromolar concentrations [46], Clostridia will also preferentially oxidise carbohydrates through higher ATP-yielding pathways and reoxidise the NAD and ferredoxin reduced using the electron-bifurcating hydrogenase [29]. It is likely that the ferredoxin-only hydrogenases are preferentially upregulated during the transient periods where $\mathrm{H}_{2}$ levels are high, for example immediately after feeding [46]. Based on these findings and previously published results [29, 66, 104, 105], we propose that the hydrogenases and fermentation pathways are differentially regulated as a result of direct $\mathrm{H}_{2}$ sensing by putative sensory [ $\mathrm{FeFe}]$-hydrogenases. A further surprising finding is that uncharacterised lineages within the Clostridia account for a large proportion of the electronbifurcating hydrogenase reads, emphasising the need for physiological and bacteriological characterisation of these organisms.

The other major finding of this work is that there are multiple highly active $\mathrm{H}_{2}$ sinks in the rumen. We provide evidence, based on transcript levels of their hydrogenases and terminal reductases, that acetogens (Blautia, Acetitomaculum), fumarate and nitrate reducers (Selenomonas, Wolinella) and sulfate reducers (Desulfovibrio) are quantitatively significant $\mathrm{H}_{2}$ sinks in sheep. In support of these findings, our culture-based study confirmed that the enzymes mediating hydrogenotrophic fumarate reduction and potentially nitrate ammonification are highly expressed by $W$. succinogenes in co-culture with $R$. albus. Although alternative $\mathrm{H}_{2}$ uptake pathways have been previously detected in vitro [41, 42, 50-53, 57, 58], it has generally been assumed that they are quantitatively insignificant compared to hydrogenotrophic methanogenesis $[5,6,46]$. To the contrary, hydrogenase and terminal reductase transcripts from alternative $\mathrm{H}_{2}$ uptake pathways are more numerous than those of methanogens in low methane yield sheep, and hence these pathways may collectively serve as a larger $\mathrm{H}_{2}$ sink than methanogenesis under some circumstances. These findings justify activity-based studies to quantify $\mathrm{H}_{2}$ flux within ruminants between methanogenic and non-methanogenic pathways. There is also evidence of other novel pathways operating in the rumen, mediated by the functionally unresolved group $1 \mathrm{i}[\mathrm{NiFe}]$-hydrogenases (Slackia, Denitrobacterium), group $4 \mathrm{~g}[\mathrm{NiFe}]$-hydrogenase (Clostridium) and group A2 [FeFe]-hydrogenases (Sharpea, Oribacterium, Olsenella).

These findings also provide some insight into the basis of variation in methane emissions between ruminants. Methane yield in ruminants is a quantitative heritable trait $[64,77]$, to some extent, and there is much interest in developing host or microbiome markers to enable selection of animals with low methane yield traits [109]. Our study compared the abundance and expression of key functional genes in the rumen microbial communities of sheep that differed by an average of $28 \%$ in methane yield. In low methane yield sheep, there was significantly decreased expression of methyl-CoM reductase, increased expression of acetyl-CoA synthase and fumarate reductase and multiple changes in hydrogenase profiles. However, we detected few significant differences at the gene level. The similar metagenome profiles, combined with the metatranscriptome profiles of the phenotype-switching sheep, therefore suggest that alternative $\mathrm{H}_{2}$ uptake pathways are partly inducible. Further studies with larger sample sizes are needed to assess whether transcripts of these functional genes can predict methane yield and, if so, whether they can be adopted 
as biomarkers to facilitate animal selection or evaluate methane mitigation strategies. Our findings, in conjunction with other studies [109], indicate that the methyl-CoM reductase and hydrogenases of methanogens may be particularly promising and relevant biomarkers.

The strong correlation between $\mathrm{H}_{2}$ uptake pathways and methane yield phenotypes nevertheless supports that modulating $\mathrm{H}_{2}$ metabolism may be an effective methane mitigation strategy. One strategy is to develop inhibitors that redirect electron flux from $\mathrm{H}_{2}$ production towards volatile fatty acid production. However, given the central role of $\mathrm{H}_{2}$ metabolism in the physiology and ecology of most rumen microorganisms, this would be challenging to achieve without compromising rumen function and consequently ruminant nutrition. Furthermore, such strategies may have a converse effect on methane production, given lower $\mathrm{H}_{2}$ concentrations restrict acetogens more than methanogens [46]. Instead, our metatranscriptome analyses support the concept that a more promising approach may be to stimulate alternative $\mathrm{H}_{2}$ pathways such as fumarate, nitrate and sulfate respiration. One solution may be to supplement animal feeds with electron acceptors, such as fumarate, nitrate, or sulfate, that stimulate the dominant respiratory hydrogenotrophs. Such approaches have shown some promise in mitigating methane production both in vitro [110-112] and in field trials $[62,63,113,114]$. These strategies may complement methanogenesis inhibitors [16, 17] by facilitating the redirection of $\mathrm{H}_{2}$ flux from methanogens to other pathways. Although further studies are required, there is also growing evidence that redirecting $\mathrm{H}_{2}$ flux can favour animal production by enabling recovery of energy that would otherwise be lost through methane emissions [115].

Acknowledgements This study was funded by the New Zealand Government to support the objectives of the Livestock Research Group of the Global Research Alliance on Agricultural Greenhouse Gases via a grant from the New Zealand Fund for Global Partnerships in Livestock Emissions Research (SOW14-GPLER4-AGR-SP6; awarded to G.T.A., S.C.L., W.J.K., R.M., S.K. and G.M.C.). Transcriptomic and metabolic research on the co-cultures was supported by the Agriculture and Food Research Initiative competitive grant 2012-67015-19451 from the USDA National Institute of Food and Agriculture (awarded to R.I.M. and I.C.). The study was also supported by an ARC DECRA Fellowship (DE170100310; awarded to C.G.), an ARC Future Fellowship (FT170100441; awarded to M.J.M.) and a PhD scholarship awarded by Monash University (L.C.W.).

Author contributions G.M.C., R.I.M., G.T.A., I.C., S.C.L., W.J.K., S. K. and C.G. conceived this study. C.G., R.I.M., G.M.C., I.C., S.E.M., M.J.M., X.C.M. and G.T.A. designed research, supervised students and analysed data. C.G., L.C.W., M.J.M. and S.C.L. performed the comparative genomic analysis. C.G., C.W., S.E.M., G.M.C., R.R.G., G.T.A., W.J.K., S.C.L. and X.C.M. performed the metagenomic and metatranscriptomic analysis. R.G. performed the co-culture experiments and R.G., R.I.M., I.C. and C.G. analysed the results. C.G. wrote and illustrated the paper with input from all authors.

\section{Compliance with ethical standards}

Conflict of interest The authors declare that they have no conflict of interest.

Publisher's note: Springer Nature remains neutral with regard to jurisdictional claims in published maps and institutional affiliations.

Open Access This article is licensed under a Creative Commons Attribution 4.0 International License, which permits use, sharing, adaptation, distribution and reproduction in any medium or format, as long as you give appropriate credit to the original author(s) and the source, provide a link to the Creative Commons license, and indicate if changes were made. The images or other third party material in this article are included in the article's Creative Commons license, unless indicated otherwise in a credit line to the material. If material is not included in the article's Creative Commons license and your intended use is not permitted by statutory regulation or exceeds the permitted use, you will need to obtain permission directly from the copyright holder. To view a copy of this license, visit http://creativecommons. org/licenses/by/4.0/.

\section{References}

1. Kirschke $\mathrm{S}$, Bousquet $\mathrm{P}$, Ciais $\mathrm{P}$, Saunois $\mathrm{M}$, Canadell JG, Dlugokencky EJ, et al. Three decades of global methane sources and sinks. Nat Geosci. 2013;6:813-23.

2. Beijer WH. Methane fermentation in the rumen of cattle. Nature. 1952;170:576.

3. Whitford MF, Teather RM, Forster RJ. Phylogenetic analysis of methanogens from the bovine rumen. BMC Microbiol. 2001;1:5.

4. Wright A-DG, Williams AJ, Winder B, Christophersen CT, Rodgers SL, Smith KD. Molecular diversity of rumen methanogens from sheep in Western Australia. Appl Environ Microbiol. 2004;70:1263-70.

5. Henderson G, Cox F, Ganesh S, Jonker A, Young W, Abecia L, et al. Rumen microbial community composition varies with diet and host, but a core microbiome is found across a wide geographical range. Sci Rep. 2015;5:14567.

6. Hungate RE. Hydrogen as an intermediate in the rumen fermentation. Arch Microbiol. 1967;59:158-64.

7. Hungate RE, Smith W, Bauchop T, Yu I, Rabinowitz JC. Formate as an intermediate in the bovine rumen fermentation. $\mathbf{J}$ Bacteriol. 1970;102:389-97.

8. Thauer RK. The Wolfe cycle comes full circle. Proc Natl Acad Sci USA. 2012;109:15084-5.

9. Leahy SC, Kelly WJ, Altermann E, Ronimus RS, Yeoman CJ, Pacheco DM, et al. The genome sequence of the rumen methanogen Methanobrevibacter ruminantium reveals new possibilities for controlling ruminant methane emissions. PLoS ONE. 2010;5:e8926.

10. Kelly WJ, Leahy SC, Li D, Perry R, Lambie SC, Attwood GT, et al. The complete genome sequence of the rumen methanogen Methanobacterium formicicum BRM9. Stand Genomic Sci. 2014;9:15.

11. Lambie SC, Kelly WJ, Leahy SC, Li D, Reilly K, McAllister $\mathrm{TA}$, et al. The complete genome sequence of the rumen methanogen Methanosarcina barkeri CM1. Stand Genomic Sci. 2015;10:57.

12. Li Y, Leahy SC, Jeyanathan J, Henderson G, Cox F, Altermann $\mathrm{E}$, et al. The complete genome sequence of the methanogenic archaeon ISO4-H5 provides insights into the methylotrophic lifestyle of a ruminal representative of the Methanomassiliicoccales. Stand Genomic Sci. 2016;11:59. 
13. Martin C, Morgavi DP, Doreau M. Methane mitigation in ruminants: from microbe to the farm scale. Animal. 2010;4:351-65.

14. Buddle BM, Denis M, Attwood GT, Altermann E, Janssen PH, Ronimus RS, et al. Strategies to reduce methane emissions from farmed ruminants grazing on pasture. Vet J. 2011;188:11-17.

15. Wedlock DN, Janssen PH, Leahy SC, Shu D, Buddle BM. Progress in the development of vaccines against rumen methanogens. Animal. 2013;7:244-52.

16. Hristov AN, Oh J, Giallongo F, Frederick TW, Harper MT, Weeks HL, et al. An inhibitor persistently decreased enteric methane emission from dairy cows with no negative effect on milk production. Proc Natl Acad Sci. 2015;112:10663-8.

17. Henderson G, Cook GM, Ronimus RS. Enzyme-and gene-based approaches for developing methanogen-specific compounds to control ruminant methane emissions: a review. Anim Prod Sci. 2018;58:1017-26.

18. Weimar MR, Cheung J, Dey D, McSweeney C, Morrison M, Kobayashi Y, et al. Development of multi-well plate methods using pure cultures of methanogens to identify new inhibitors for suppressing ruminant methane emissions. Appl Environ Microbiol. 2017;83:e00396-17.

19. Morgavi DP, Forano E, Martin C, Newbold CJ. Microbial ecosystem and methanogenesis in ruminants. Animal. 2010;4:1024-36.

20. Malik PK, Bhatta R, Gagen EJ, Sejian V, Soren NM, Prasad CS. Alternate $\mathrm{H}_{2}$ Sinks for Reducing Rumen Methanogenesis. In: Climate change impact on livestock: adaptation and mitigation 2015. Springer, 303-20.

21. Seshadri R, Leahy SC, Attwood GT, Teh KH, Lambie SC, Cookson $\mathrm{AL}$, et al. Cultivation and sequencing of rumen microbiome members from the Hungate1000 Collection. Nat Biotechnol. 2018;36:359.

22. Solden LM, Naas AE, Roux S, Daly RA, Collins WB, Nicora $\mathrm{CD}$, et al. Interspecies cross-feeding orchestrates carbon degradation in the rumen ecosystem. Nat Microbiol. 2018;3:1274.

23. Stewart RD, Auffret MD, Warr A, Wiser AH, Press MO, Langford KW, et al. Assembly of 913 microbial genomes from metagenomic sequencing of the cow rumen. Nat Commun. 2018;9:870.

24. Miller TL, Wolin MJ. Formation of hydrogen and formate by Ruminococcus albus. J Bacteriol. 1973;116:836-46.

25. Iannotti EL, Kafkewitz D, Wolin MJ, Bryant MP. Glucose fermentation products of Ruminococcus albus grown in continuous culture with Vibrio succinogenes: changes caused by interspecies transfer of $\mathrm{H}_{2}$. J Bacteriol. 1973;114:1231-40.

26. Varel VH. Reisolation and characterization of Clostridium longisporum, a ruminal sporeforming cellulolytic anaerobe. Arch Microbiol. 1989;152:209-14.

27. Scheifinger $\mathrm{CC}$, Linehan $\mathrm{B}$, Wolin $\mathrm{MJ} . \mathrm{H}_{2}$ production by Selenomonas ruminantium in the absence and presence of methanogenic bacteria. Appl Microbiol. 1975;29:480-3.

28. Hino T, Miyazaki K, Kuroda S. Role of extracellular acetate in the fermentation of glucose by a ruminal bacterium, Megasphaera elsdenii. J Gen Appl Microbiol. 1991;37:121-9.

29. Zheng Y, Kahnt J, Kwon IH, Mackie RI, Thauer RK. Hydrogen formation and its regulation in Ruminococcus albus: involvement of an electron-bifurcating [FeFe]-hydrogenase, of a non-electron-bifurcating $[\mathrm{FeFe}]$-hydrogenase, and of a putative hydrogen-sensing [FeFe]-hydrogenase. J Bacteriol. 2014; 196:3840-52.

30. Gutierrez J, Davis RE. Culture and metabolism of the rumen ciliate Epidinium ecaudatum Crawley. Appl Microbiol. 1962;10:305-8.

31. Bauchop T, Mountfort DO. Cellulose fermentation by a rumen anaerobic fungus in both the absence and the presence of rumen methanogens. Appl Environ Microbiol. 1981;42:1103-10.

32. Li Pun HH, Satter LD. Nitrogen fixation in ruminants. J Anim Sci. 1975;41:1161-3.
33. Granhall U, Ciszuk P. Nitrogen fixation in rumen contents indicated by the acetylene reduction test. Microbiology. 1971;65:91-93.

34. Latham MJ, Wolin MJ. Fermentation of cellulose by Ruminococcus flavefaciens in the presence and absence of Methanobacterium ruminantium. Appl Environ Microbiol. 1977;34:297-301.

35. Vogels GD, Hoppe WF, Stumm CK. Association of methanogenic bacteria with rumen ciliates. Appl Environ Microbiol. 1980;40:608-12.

36. Finlay BJ, Esteban G, Clarke KJ, Williams AG, Embley TM, Hirt RP. Some rumen ciliates have endosymbiotic methanogens. FEMS Microbiol Lett. 1994;117:157-61.

37. Ng F, Kittelmann S, Patchett ML, Attwood GT, Janssen PH, Rakonjac J, et al. An adhesin from hydrogen-utilizing rumen methanogen Methanobrevibacter ruminantium M 1 binds a broad range of hydrogen-producing microorganisms. Environ Microbiol. 2016;18:3010-21.

38. Stams AJM, Plugge CM. Electron transfer in syntrophic communities of anaerobic bacteria and archaea. Nat Rev Microbiol. 2009;7:568.

39. Schuchmann K, Muller V. Autotrophy at the thermodynamic limit of life: a model for energy conservation in acetogenic bacteria. Nat Rev Microbiol. 2014;12:809-21.

40. Genthner BR, Davis CL, Bryant MP. Features of rumen and sewage sludge strains of Eubacterium limosum, a methanol-and $\mathrm{H}_{2}-\mathrm{CO}_{2}$-utilizing species. Appl Environ Microbiol. 1981;42:12-19.

41. Rieu-Lesme F, Morvan B, Collins MD, Fonty G, Willems A. A new $\mathrm{H}_{2} / \mathrm{CO}_{2}$-using acetogenic bacterium from the rumen: description of Ruminococcus schinkii sp. nov. FEMS Microbiol Lett. 1996;140:281-6.

42. Greening RC, Leedle JAZ. Enrichment and isolation of Acetitomaculum ruminis, gen. nov., sp. nov.: acetogenic bacteria from the bovine rumen. Arch Microbiol. 1989;151:399-406.

43. Morvan B, Dore J, Rieu-Lesme F, Foucat L, Fonty G, Gouet P. Establishment of hydrogen-utilizing bacteria in the rumen of the newborn lamb. FEMS Microbiol Lett. 1994;117:249-56.

44. Morvan B, Bonnemoy F, Fonty G, Gouet P. Quantitative determination of $\mathrm{H}_{2}$-utilizing acetogenic and sulfate-reducing bacteria and methanogenic archaea from digestive tract of different mammals. Curr Microbiol. 1996;32:129-33.

45. Henderson G, Naylor GE, Leahy SC, Janssen PH. Presence of novel, potentially homoacetogenic bacteria in the rumen as determined by analysis of formyltetrahydrofolate synthetase sequences from ruminants. Appl Environ Microbiol. 2010;76:2058-66.

46. Janssen PH. Influence of hydrogen on rumen methane formation and fermentation balances through microbial growth kinetics and fermentation thermodynamics. Anim Feed Sci Technol. 2010;160:1-22.

47. Cord-Ruwisch R, Seitz H-J, Conrad R. The capacity of hydrogenotrophic anaerobic bacteria to compete for traces of hydrogen depends on the redox potential of the terminal electron acceptor. Arch Microbiol. 1988;149:350-7.

48. Joblin KN. Ruminal acetogens and their potential to lower ruminant methane emissions. Aust J Agric Res. 1999;50:1307-14.

49. Attwood G, McSweeney C. Methanogen genomics to discover targets for methane mitigation technologies and options for alternative $\mathrm{H}_{2}$ utilisation in the rumen. Aust $\mathrm{J}$ Exp Agric. 2008;48:28-37.

50. Huisingh J, McNeill JJ, Matrone G. Sulfate reduction by a Desulfovibrio species isolated from sheep rumen. Appl Microbiol. 1974;28:489-97.

51. Howard BH, Hungate RE. Desulfovibrio of the sheep rumen. Appl Environ Microbiol. 1976;32:598-602.

52. Henderson $\mathrm{C}$. The influence of extracellular hydrogen on the metabolism of Bacteroides ruminicola, Anaerovibrio lipolytica and Selenomonas ruminantium. Microbiology. 1980;119:485-91. 
53. Martin SA, Park C-M. Effect of extracellular hydrogen on organic acid utilization by the ruminal bacterium Selenomonas ruminantium. Curr Microbiol. 1996;32:327-31.

54. Wolin MJ, Wolin EA, Jacobs NJ. Cytochrome-producing anaerobic vibrio, Vibrio succinogenes, sp. n. J Bacteriol. 1961;81:911.

55. Tanner ACR, Badger S, Lai C-H. Listgarten MAXA, Visconti RA, Socransky SS. Wolinella gen. nov., Wolinella succinogenes (Vibrio succinogenes Wolin et al.) comb. nov., and description of Bacteroides gracilis sp. nov., Wolinella recta $\mathrm{sp}$. nov., <i> Campylobacter concisus sp. nov. Int J Syst Evol Microbiol. 1981;31:432-45.

56. Unden $\mathrm{G}$, Hackenberg $\mathrm{H}$, Kröger A. Isolation and functional aspects of the fumarate reductase involved in the phosphorylative electron transport of Vibrio succinogenes. Biochim Biophys Acta. 1980;591:275-88.

57. Jones GA. Dissimilatory metabolism of nitrate by the rumen microbiota. Can J Microbiol. 1972;18:1783-7.

58. Iwamoto M, Asanuma N, Hino T. Ability of Selenomonas ruminantium, Veillonella parvula, and Wolinella succinogenes to reduce nitrate and nitrite with special reference to the suppression of ruminal methanogenesis. Anaerobe. 2002;8:209-15.

59. Bokranz M, Katz J, Schröder I, Roberton AM, Kröger A. Energy metabolism and biosynthesis of Vibrio succinogenes growing with nitrate or nitrite as terminal electron acceptor. Arch Microbiol. 1983;135:36-41.

60. Anderson RC, Rasmussen MA, Jensen NS, Allison MJ. Denitrobacterium detoxificans gen. nov., sp. nov., a ruminal bacterium that respires on nitrocompounds. Int J Syst Evol Microbiol. 2000;50:633-8.

61. Schwartz E, Fritsch J, Friedrich B. H2-metabolizing prokaryotes. Berlin, Heidelberg: Springer; 2013.

62. Van Zijderveld SM, Gerrits WJJ, Apajalahti JA, Newbold JR, Dijkstra J, Leng RA, et al. Nitrate and sulfate: effective alternative hydrogen sinks for mitigation of ruminal methane production in sheep. J Dairy Sci. 2010;93:5856-66.

63. Bayaru E, Kanda S, Kamada T, Itabashi H, Andoh S, Nishida T, et al. Effect of fumaric acid on methane production, rumen fermentation and digestibility of cattle fed roughage alone. Nihon Chikusan Gakkaiho. 2001;72:139-46.

64. Shi W, Moon CD, Leahy SC, Kang D, Froula J, Kittelmann S, et al. Methane yield phenotypes linked to differential gene expression in the sheep rumen microbiome. Genome Res. 2014;24:1517-25.

65. Kamke J, Kittelmann S, Soni P, Li Y, Tavendale M, Ganesh S, et al. Rumen metagenome and metatranscriptome analyses of low methane yield sheep reveals a Sharpea-enriched microbiome characterised by lactic acid formation and utilisation. Microbiome. 2016;4:56.

66. Greening C, Biswas A, Carere CR, Jackson CJ, Taylor MC, Stott $\mathrm{MB}$, et al. Genomic and metagenomic surveys of hydrogenase distribution indicate $\mathrm{H} 2$ is a widely utilised energy source for microbial growth and survival. ISME J. 2016;10:761-77.

67. Søndergaard D, Pedersen CNS, Greening C. HydDB: a web tool for hydrogenase classification and analysis. Sci Rep. 2016;6:34212.

68. Bryant MP, Small N, Bouma C, Robinson IM. Characteristics of ruminal anaerobic cellulolytic cocci and Cillobacterium cellulosolvens n. sp. J Bacteriol. 1958;76:529.

69. Kittelmann S, Pinares-Patiño CS, Seedorf H, Kirk MR, Ganesh S, McEwan JC, et al. Two different bacterial community types are linked with the low-methane emission trait in sheep. PLoS ONE. 2014;9:e103171.

70. Evans PN, Parks DH, Chadwick GL, Robbins SJ, Orphan VJ, Golding SD, et al. Methane metabolism in the archaeal phylum Bathyarchaeota revealed by genome-centric metagenomics. Science. 2015;350:434-8.
71. Adam PS, Borrel G, Gribaldo S. Evolutionary history of carbon monoxide dehydrogenase/acetyl-CoA synthase, one of the oldest enzymatic complexes. Proc Natl Acad Sci. 2018;115:201716667.

72. Anantharaman K, Hausmann B, Jungbluth SP, Kantor RS, Lavy A, Warren LA, et al. Expanded diversity of microbial groups that shape the dissimilatory sulfur cycle. ISME J. 2018;1:1715-28.

73. Lemos RS, Fernandes AS, Pereira MM, Gomes CM, Teixeira M. Quinol: fumarate oxidoreductases and succinate: quinone oxidoreductases: phylogenetic relationships, metal centres and membrane attachment. Biochim Biophys Acta. 2002;1553:158-70.

74. Boyd E, Peters JW. New insights into the evolutionary history of biological nitrogen fixation. Front Microbiol. 2013;4:201.

75. Cock PJA, Antao T, Chang JT, Chapman BA, Cox CJ, Dalke A, et al. Biopython: freely available Python tools for computational molecular biology and bioinformatics. Bioinformatics. 2009;25:1422-3.

76. Altschul SF, Gish W, Miller W, Myers EW, Lipman DJ. Basic local alignment search tool. J Mol Biol. 1990;215:403-10.

77. Pinares-Patiño CS, Hickey SM, Young EA, Dodds KG, MacLean S, Molano G, et al. Heritability estimates of methane emissions from sheep. Animal. 2013;7:316-21.

78. Buchfink B, Xie C, Huson DH. Fast and sensitive protein alignment using DIAMOND. Nat Methods. 2014;12:59.

79. Balch WE, Fox GE, Magrum LJ, Woese CR, Wolfe RS. Methanogens: reevaluation of a unique biological group. Microbiol Rev. 1979;43:260.

80. Suen G, Stevenson DM, Bruce DC, Chertkov O, Copeland A, Cheng J-F, et al. Complete genome of the cellulolytic ruminal bacterium Ruminococcus albus 7. J Bacteriol. 2011;193:5574-5.

81. Baar C, Eppinger M, Raddatz G, Simon J, Lanz C, Klimmek O, et al. Complete genome sequence and analysis of Wolinella succinogenes. Proc Natl Acad Sci. 2003;100:11690-5.

82. Baggerly KA, Deng L, Morris JS, Aldaz CM. Differential expression in SAGE: accounting for normal between-library variation. Bioinformatics. 2003;19:1477-83.

83. Storey JD, Tibshirani R. Statistical significance for genomewide studies. Proc Natl Acad Sci. 2003;100:9440-5.

84. Aziz RK, Bartels D, Best AA, DeJongh M, Disz T, Edwards RA, et al. The RAST Server: rapid annotations using subsystems technology. BMC Genomics. 2008;9:75.

85. Carere CR, Hards K, Houghton KM, Power JF, McDonald B, Collet $\mathrm{C}$, et al. Mixotrophy drives niche expansion of verrucomicrobial methanotrophs. ISME J. 2017;11:2599-10.

86. Ji M, Greening C, Vanwonterghem I, Carere CR, Bay SK, Steen $\mathrm{JA}$, et al. Atmospheric trace gases support primary production in Antarctic desert surface soil. Nature. 2017;552:400-3.

87. Dong X, Greening C, Brüls T, Conrad R, Guo K, Blaskowski $\mathrm{S}$, et al. Fermentative Spirochaetes mediate necromass recycling in anoxic hydrocarbon-contaminated habitats. ISME J. 2018;12:2039-50.

88. Dyksma S, Pjevac P, Ovanesov K, Mussmann M. Evidence for $\mathrm{H}_{2}$ consumption by uncultured Desulfobacterales in coastal sediments. Environ Microbiol. 2018;20:450-61.

89. Kessler AJ, Chen Y-J, Waite DW, Hutchinson T, Koh S, Popa ME et al. Bacterial fermentation and respiration processes are uncoupled in permeable sediments. Nat Microbiol. 2019;4:1014-23.

90. Peters JW, Lanzilotta WN, Lemon BJ, Seefeldt LC. X-ray crystal structure of the Fe-only hydrogenase $(\mathrm{CpI})$ from Clostridium pasteurianum to 1.8 Angstrom resolution. Science. 1998;282:1853-8.

91. Betian HG, Linehan BA, Bryant MP, Holdeman LV. Isolation of a cellulolytic Bacteroides sp. from human feces. Appl Environ Microbiol. 1977;33:1009-10.

92. Biswas R, Zheng T, Olson DG, Lynd LR, Guss AM. Elimination of hydrogenase active site assembly blocks $\mathrm{H}_{2}$ production and increases ethanol yield in Clostridium thermocellum. Biotechnol Biofuels. 2015;8:20. 
93. Schut GJ, Adams MWW. The iron-hydrogenase of Thermotoga maritima utilizes ferredoxin and NADH synergistically: a new perspective on anaerobic hydrogen production. J Bacteriol. 2009;191:4451-7.

94. Schuchmann K, Müller V. A bacterial electron-bifurcating hydrogenase. J Biol Chem. 2012;287:31165-71.

95. Vignais PM, Billoud B. Occurrence, classification, and biological function of hydrogenases: an overview. Chem Rev. 2007; 107:4206-72.

96. Jones K, Thomas JG. Nitrogen fixation by the rumen contents of sheep. Microbiology. 1974;85:97-101.

97. Chen S, Niu L, Zhang Y. Saccharofermentans acetigenes gen. nov., sp. nov., an anaerobic bacterium isolated from sludge treating brewery wastewater. Int J Syst Evol Microbiol. 2010;60:2735-8.

98. Allegretti M, Mills DJ, McMullan G, Kühlbrandt W, Vonck J. Atomic model of the $\mathrm{F}_{420}$-reducing $[\mathrm{NiFe}]$ hydrogenase by electron cryo-microscopy using a direct electron detector. Elife. 2014;3:e01963.

99. Wagner T, Koch J, Ermler U, Shima S. Methanogenic heterodisulfide reductase (HdrABC-MvhAGD) uses two noncubane [4Fe-4S] clusters for reduction. Science. 2017;357:699-703.

100. Lie TJ, Costa KC, Lupa B, Korpole S, Whitman WB, Leigh JA. Essential anaplerotic role for the energy-converting hydrogenase Eha in hydrogenotrophic methanogenesis. Proc Natl Acad Sci USA. 2012;109:15473-8.

101. Major TA, Liu Y, Whitman WB. Characterization of energyconserving hydrogenase $\mathrm{B}$ in Methanococcus maripaludis. J Bacteriol. 2010;192:4022-30.

102. Shima S, Pilak O, Vogt S, Schick M, Stagni MS, Meyer-Klaucke $\mathrm{W}$, et al. The crystal structure of [Fe]-Hydrogenase reveals the geometry of the active site. Science. 2008;321:572-5.

103. Marais JP, Therion JJ, Mackie RI, Kistner A, Dennison C. Effect of nitrate and its reduction products on the growth and activity of the rumen microbial population. Br J Nutr. 1988;59:301-13.

104. Poudel S, Tokmina-Lukaszewska M, Colman DR, Refai M, Schut GJ, King PW et al. Unification of [FeFe]-hydrogenases into three structural and functional groups. Biochim Biophys Acta. 2016;1860:1910-21.

105. Chongdar N, Birrell JA, Pawlak K, Sommer C, Reijerse EJ, Rüdiger $\mathrm{O}$, et al. Unique spectroscopic properties of the $\mathrm{H}$ -
Cluster in a putative sensory $[\mathrm{FeFe}]$ hydrogenase. $\mathrm{J}$ Am Chem Soc. 2018;140:1057-68.

106. Greening C, Cook GM. Integration of hydrogenase expression and hydrogen sensing in bacterial cell physiology. Curr Opin Microbiol. 2014;18:30-8.

107. Lenz O, Friedrich B. A novel multicomponent regulatory system mediates $\mathrm{H}_{2}$ sensing in Alcaligenes eutrophus. Proc Natl Acad Sci USA. 1998;95:12474-9.

108. Stewart RD, Auffret MD, Warr A, Walker AW, Roehe R, Watson $\mathrm{M}$. The genomic and proteomic landscape of the rumen microbiome revealed by comprehensive genome-resolved metagenomics. bioRxiv 2018; 489443.

109. Auffret MD, Stewart R, Dewhurst RJ, Duthie C-A, Rooke JA, Wallace RJ, et al. Identification, comparison, and validation of robust Rumen microbial biomarkers for methane emissions using diverse Bos taurus breeds and basal diets. Front Microbiol. 2018;8:2642.

110. Asanuma N, Iwamoto M, Hino T. Effect of the addition of fumarate on methane production by ruminal microorganisms in vitro. J Dairy Sci. 1999;82:780-7.

111. Patra AK, Yu Z. Combinations of nitrate, saponin, and sulfate additively reduce methane production by rumen cultures in vitro while not adversely affecting feed digestion, fermentation or microbial communities. Bioresour Technol. 2014; 155:129-35.

112. Božic AK, Anderson RC, Carstens GE, Ricke SC, Callaway TR, Yokoyama MT, et al. Effects of the methane-inhibitors nitrate, nitroethane, lauric acid, Lauricidin ${ }^{\circledR}$ and the Hawaiian marine algae Chaetoceros on ruminal fermentation in vitro. Bioresour Technol. 2009;100:4017-25.

113. Van Zijderveld SM, Gerrits WJJ, Dijkstra J, Newbold JR, Hulshof RBA, Perdok HB. Persistency of methane mitigation by dietary nitrate supplementation in dairy cows. J Dairy Sci. 2011;94:4028-38.

114. Hulshof RBA, Berndt A, Gerrits WJJ, Dijkstra J, Van Zijderveld SM, Newbold JR, et al. Dietary nitrate supplementation reduces methane emission in beef cattle fed sugarcane-based diets. J Anim Sci. 2012;90:2317-23.

115. Ungerfeld, EM. Inhibition of rumen methanogenesis and ruminant productivity: a meta-analysis. Front Vet Sci. 2018;5:113. 